\title{
A reporter system for cytosolic protein aggregates in yeast
}

\author{
David Romero-Suarez ${ }^{1}$, Tune Wulff' ${ }^{1}$, Yixin Rong ${ }^{1}$, Tadas Jakočiūnas ${ }^{1}$, Satoshi Yuzawa2-3, \\ Jay D. Keasling ${ }^{1-5}$, Michael K. Jensen ${ }^{1 *}$ \\ ${ }^{1}$ Novo Nordisk Foundation Center for Biosustainability, Technical University of Denmark, \\ Kgs. Lyngby, Denmark \\ 2 Joint BioEnergy Institute, Emeryville, CA, USA \\ ${ }^{3}$ Biological Systems and Engineering Division, Lawrence Berkeley National Laboratory, \\ Berkeley, CA, USA \\ ${ }^{4}$ Department of Chemical and Biomolecular Engineering, Department of Bioengineering, \\ University of California, \\ Berkeley, CA, USA \\ ${ }^{5}$ Center for Synthetic Biochemistry, Institute for Synthetic Biology, Shenzhen Institutes of \\ Advanced Technologies, \\ Shenzhen, China
}

\section{Supporting Information}


Supplementary Figure 1. Quantification of bait proteins mKate2, mKate2-MBP and mKate2-LipPks1 expression by targeted proteomics. A-C) Peptide areas normalized to internal standard synthetic peptides for A) mKate2, B) MBP and C) LipPks1. D-F) Peptide areas corresponding to synthetic peptides for D) mKate2, E) MBP and F) LipPks1. n=3-4. Bars represent the mean, and error bars the standard deviation of all data points.

A

D
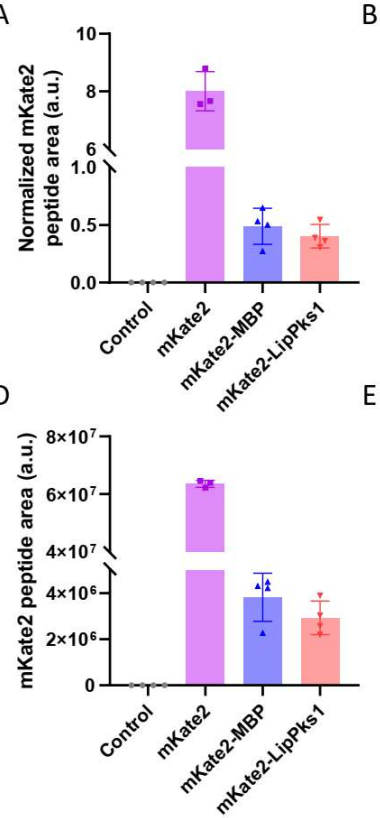
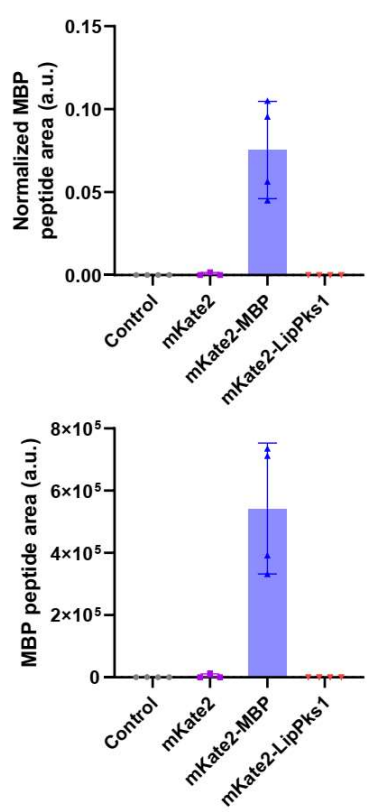

$\mathrm{F}$

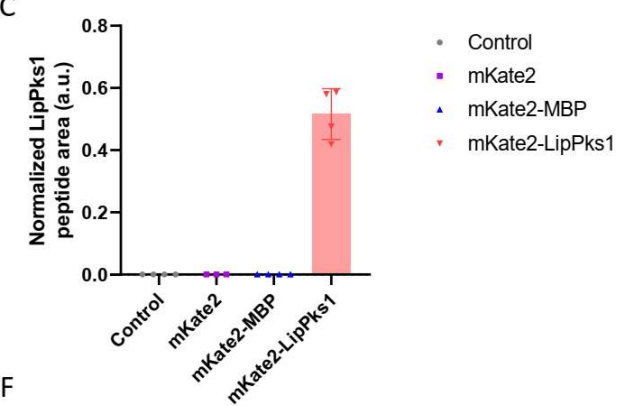

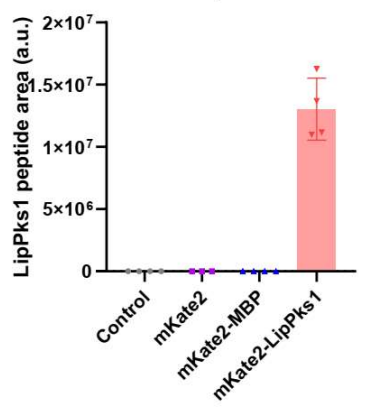


Supplementary Figure 2. Gene ontology enrichment analysis upon mKate2-LipPks1 expression. Gene ontology enrichment analysis of biological processes overrepresented in upregulated genes in response to mKate2-LipPks1 expression (Supplementary Data File 2). First 25 terms are shown.

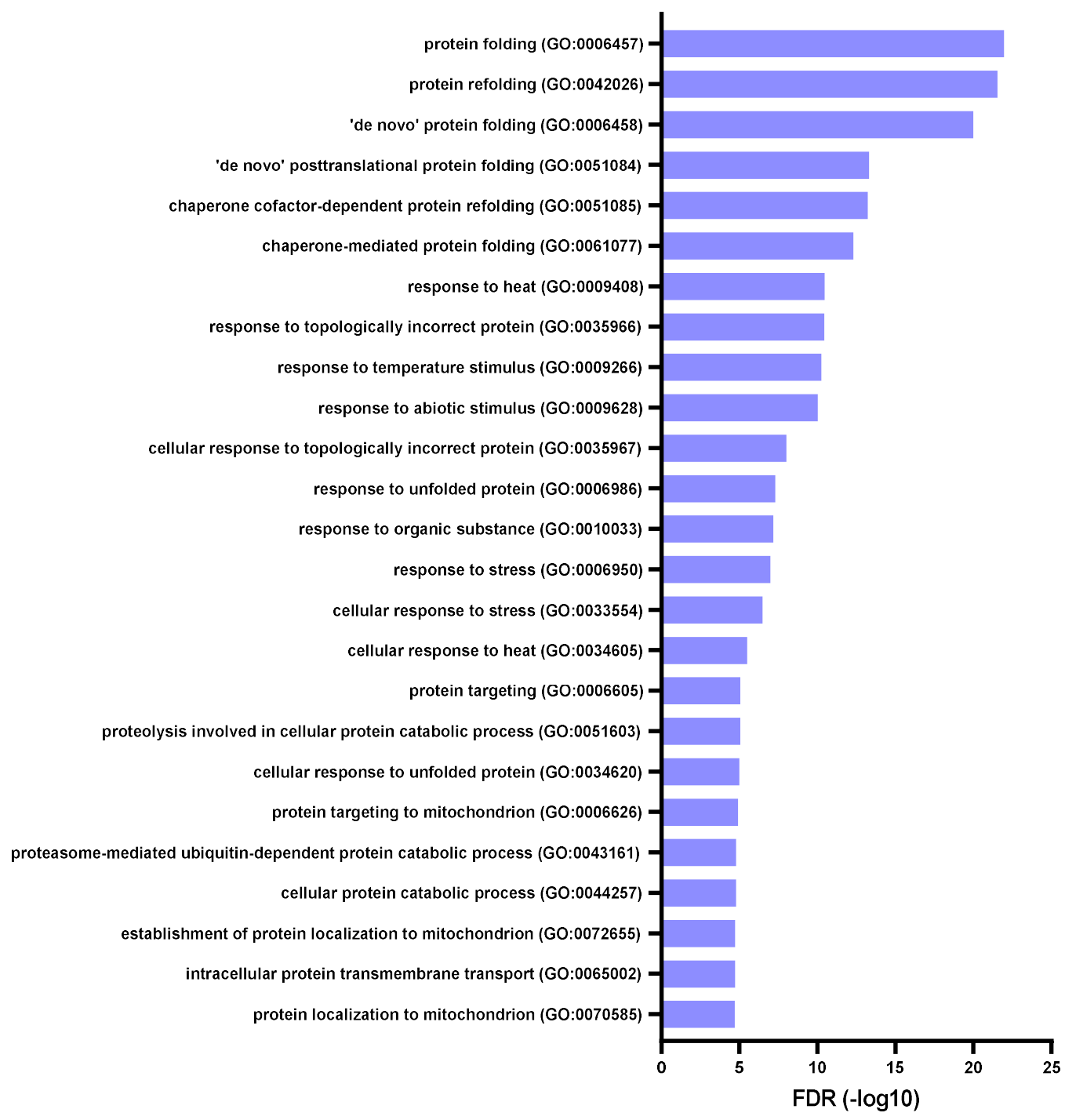


Supplementary Figure 3. Comparison of proteome responses to protein baits. Proteomes of strains expressing the protein baits mKate2, mKate2-MBP and mKate2-LipPks1 and control (no heterologous protein) were compared for differential expression ( $p \leq 0.01, F C$ 21.15). A) mKate2 vs control. B) mKate2-MBP vs control. C) mKate2-LipPks1 vs control. D) mKate2-MBP vs mKate2 E) mKate2-LipPks1 vs mKate2. F) mKate2-LipPks1 vs his6-mKate2MBP.

A

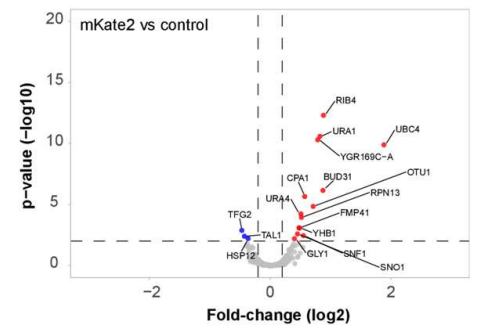

B

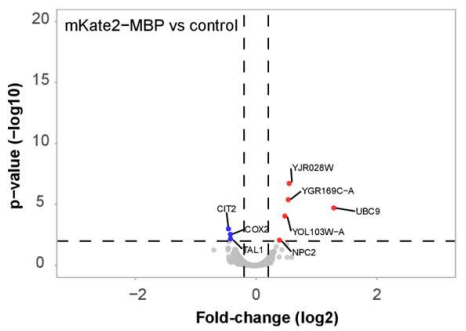

D

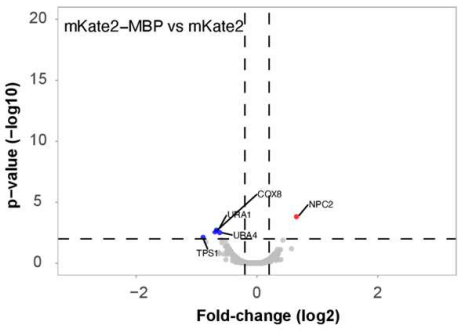

C

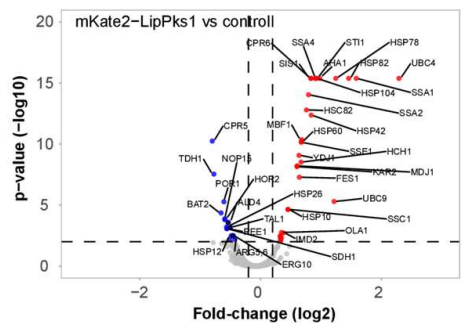

E

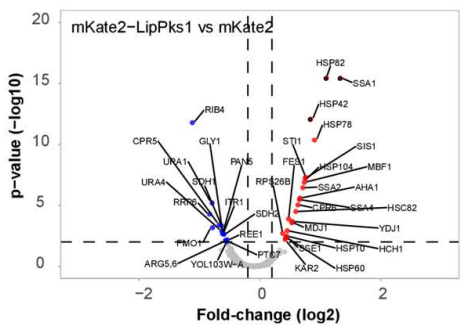

$\mathbf{F}$

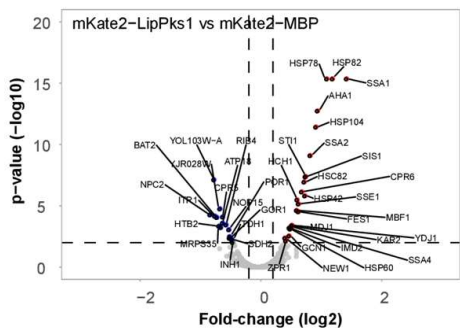

Supplementary Figure 4. Synthetic HSE-GALp1-231 reporters designs. Designs of promoters based on GAL1p core promoter (GAL1p1-231) and 1x, 2x, 4x and 8x HSE.

1XHSE-GAL1p

2XHSE-GAL1p-231

4XHSE-GAL1p

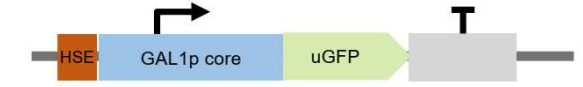

$\mathbf{T}$

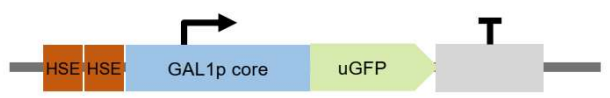

$\mathbf{T}$

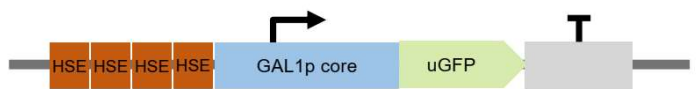


Supplementary Figure 5. Growth curves of parental strains of strains used to screen promoter-reporters. Strains expressing mKate2-MBP, mKate2-LipPks1 and control strain were grown overnight and diluted the next day in MM Medium +Ura+His+Leu+Trp. OD630 was recorded for $24 \mathrm{~h}(\mathrm{n}=6)$. Dotted line indicates $6 \mathrm{~h}$ growth. Symbols represent mean values and bars represent SD of data points.

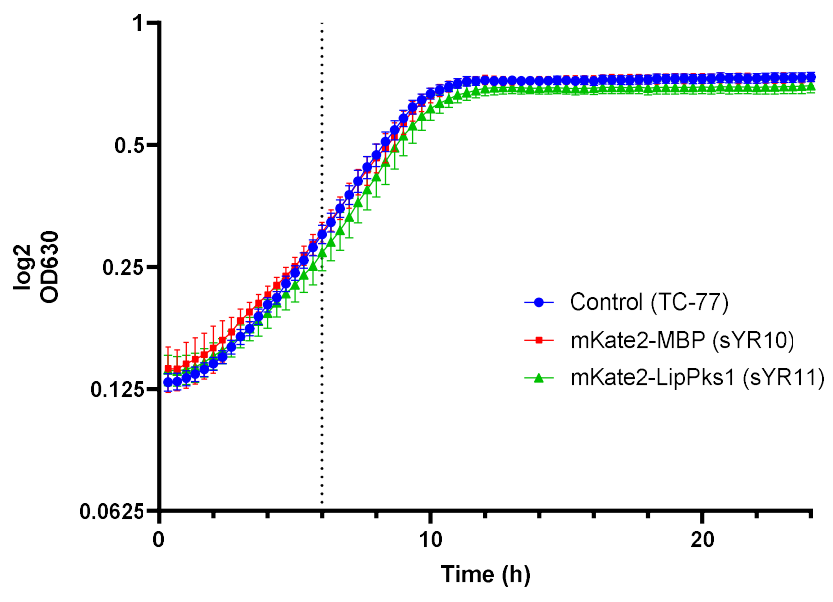


Supplementary Figure 6. Flow cytometry histograms of screen of native and synthetic promoters as aggregated protein reporters. Fluorescence distributions correspond to all events recorded for Figure 2B.
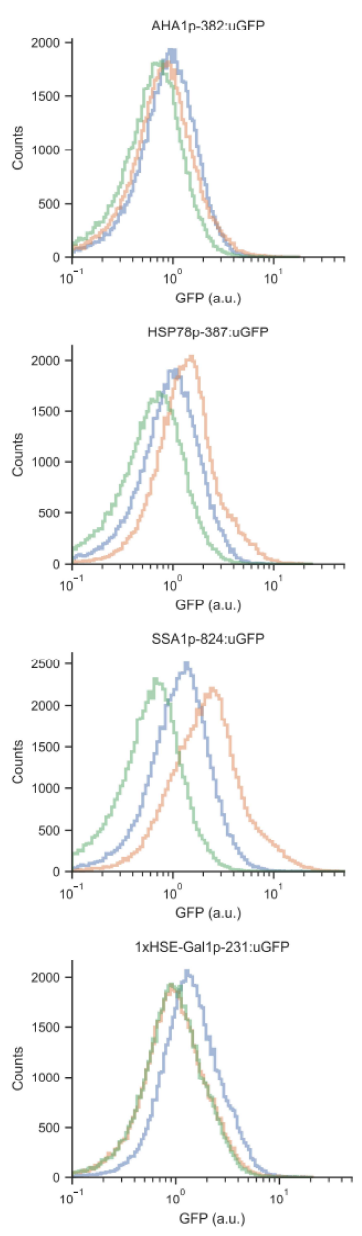
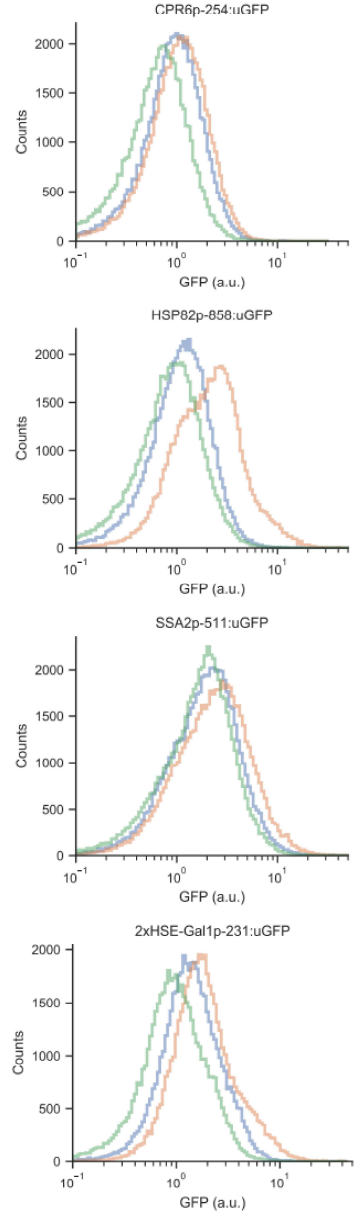
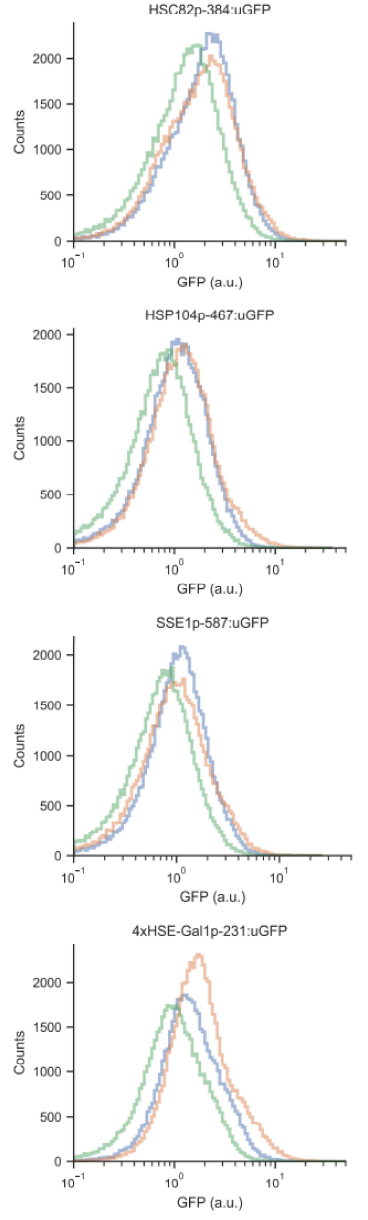
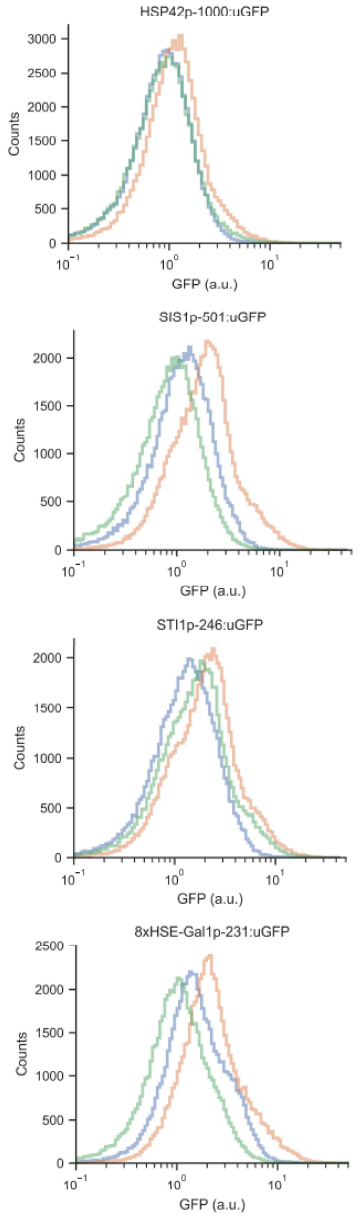

1. mKate2-MBP

2. mKate2-LipPks1

3. Only-reporter 
Supplementary Figure 7. Localization of protein baits in cellular compartments. Microscope images of strains expressing Hsp104-GFP and A) mKate2-MBP and B) mKate2LipPks1 protein baits. Cultures were grown for $6 \mathrm{~h}$ and $16 \mathrm{~h}$, with or without $50 \mu \mathrm{M}$ Bortezomib (Btz) in Mineral Medium (MM). Images were taken with different exposure times. Fluorescence intensities are not directly comparable between images.

A

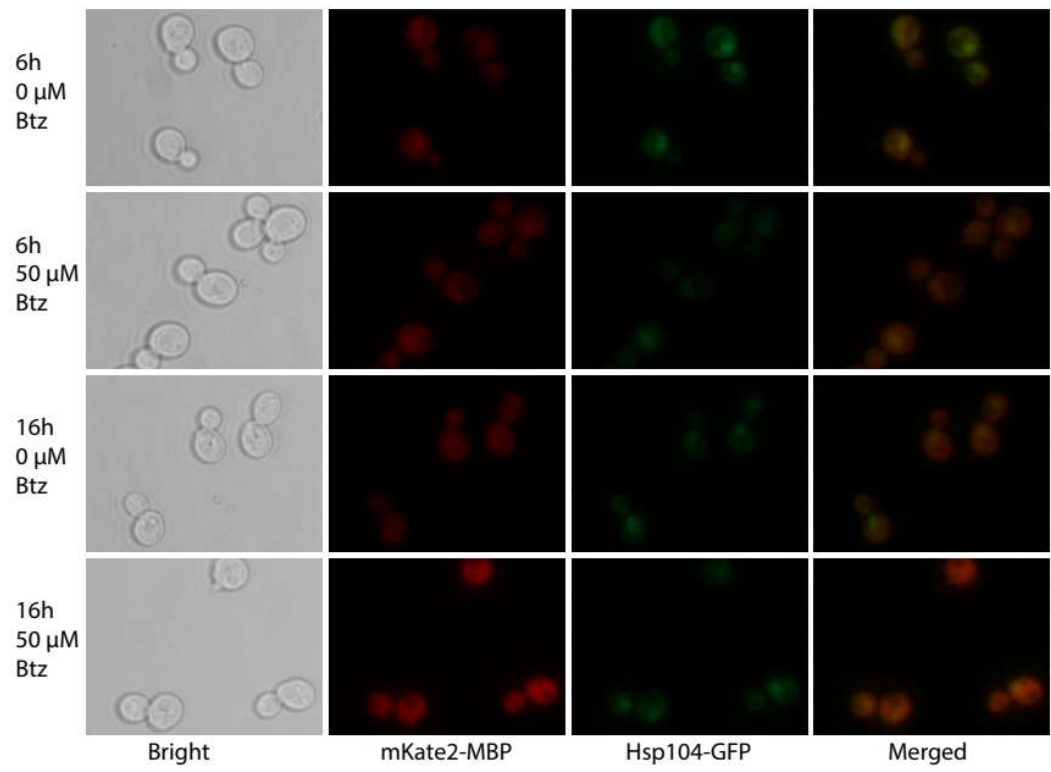

B

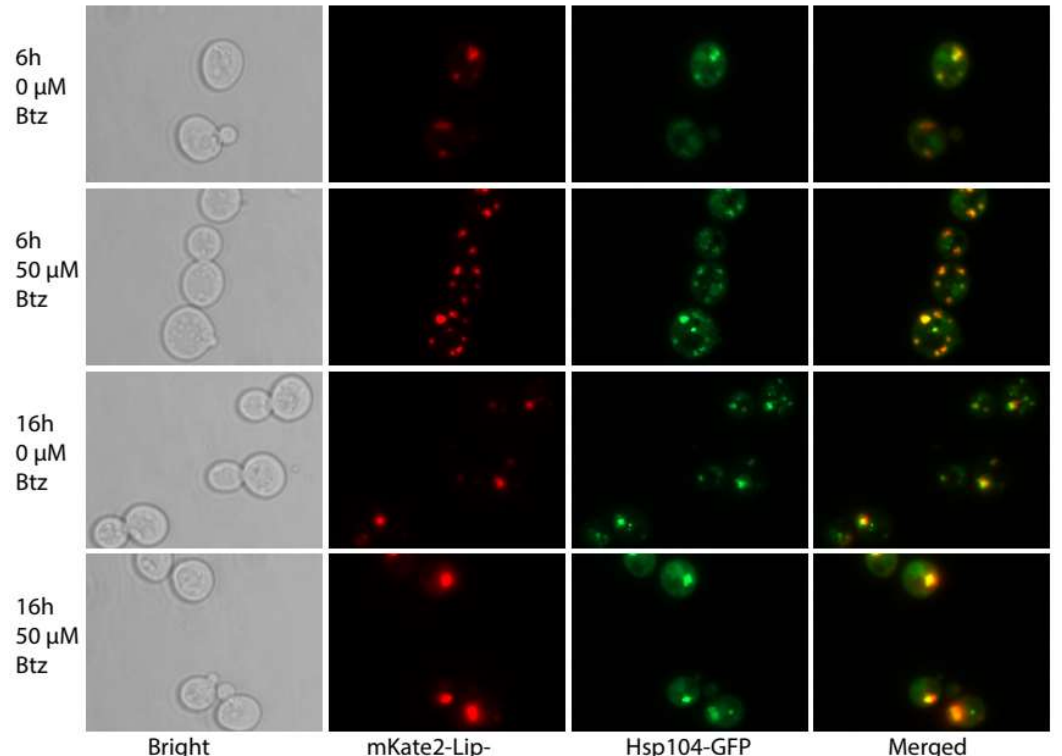


Supplementary Figure 8. Flow cytometry histograms of reporters optimization by truncations, UAS copy numbers, and GFP variants. Fluorescence distributions correspond to all events recorded for Figure 3A.
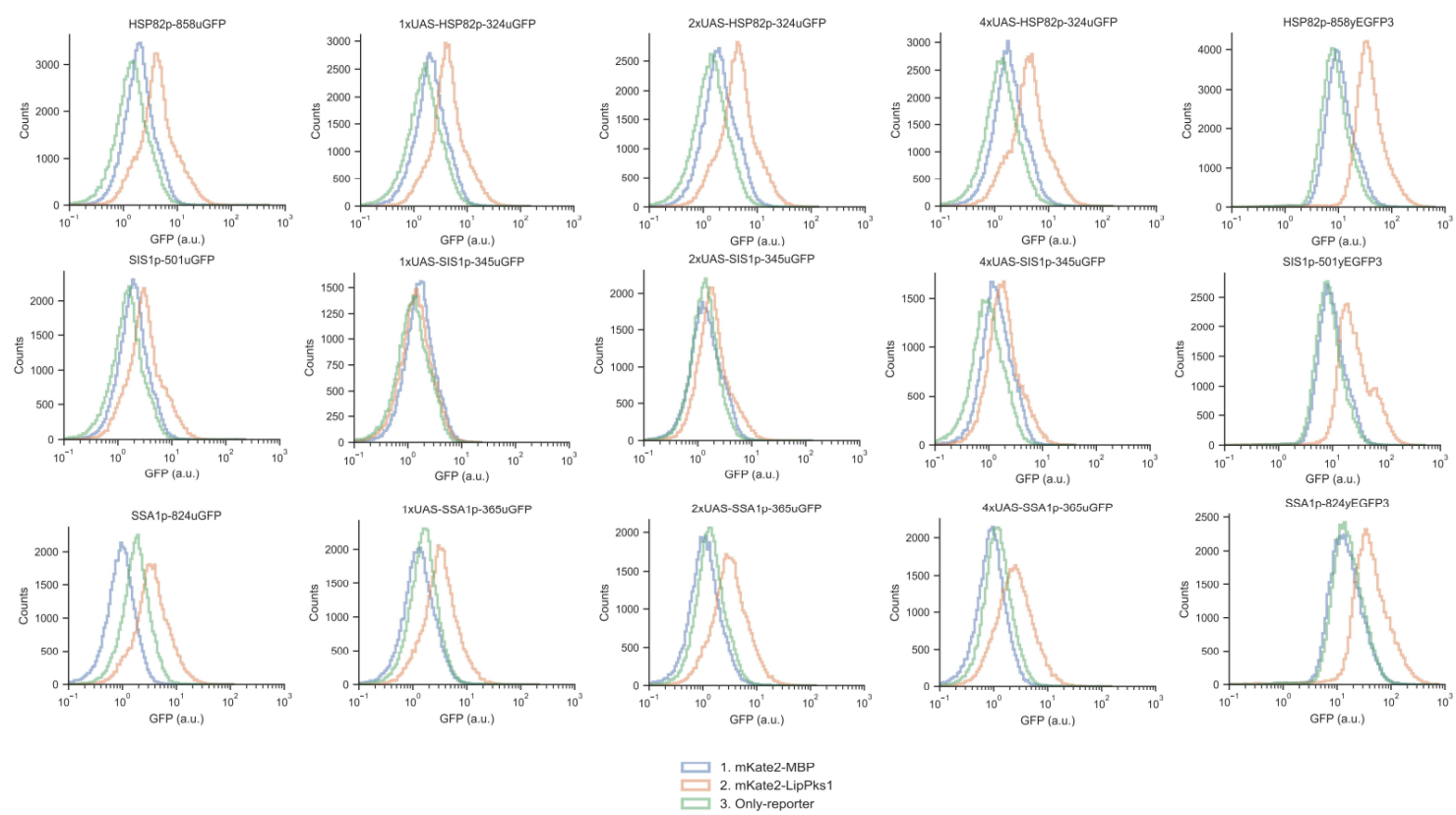
Supplementary Figure 9. Flow cytometry histograms of yEGFP3 reporters optimization by UAS copy numbers, sampling time, and proteasome inhibition. A) Promoters derived from HSP82p. B) Promoters derived from SSA1p. Fluorescence distributions correspond to all events recorded for Figure 3B.

A
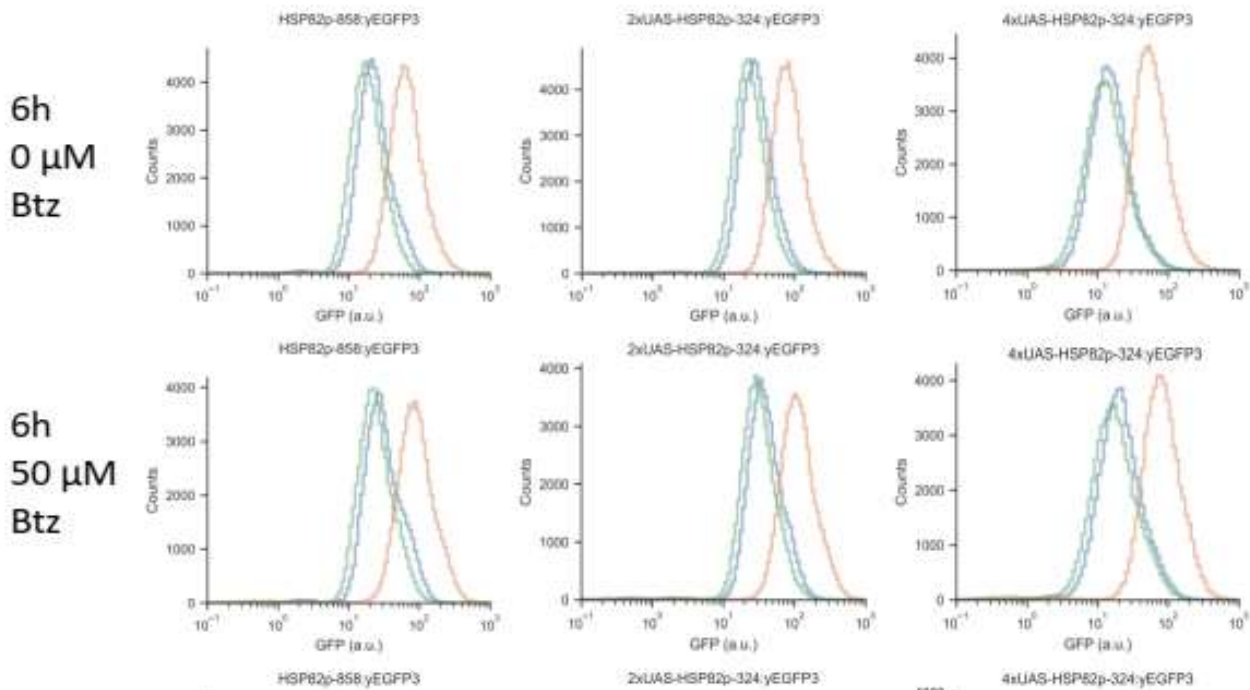

$16 \mathrm{~h}$

$0 \mu \mathrm{M}$
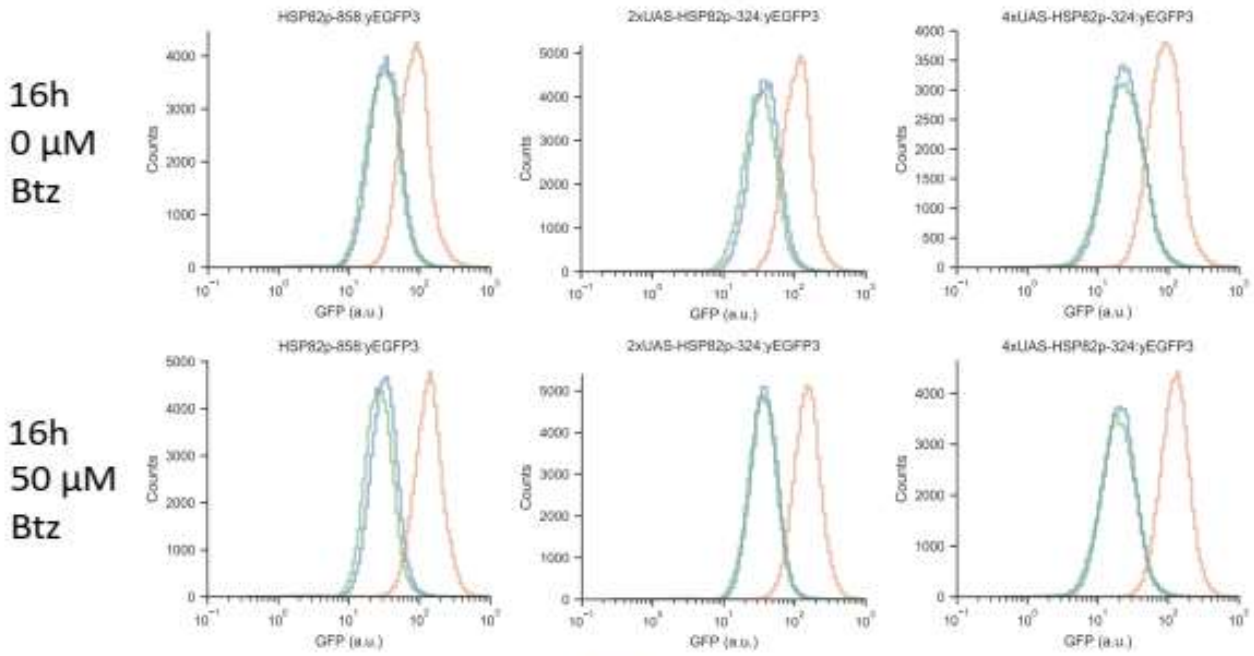

1. mKate2-MBP

2. mKate2-LipPks1

] 3. Only-reporter 
B
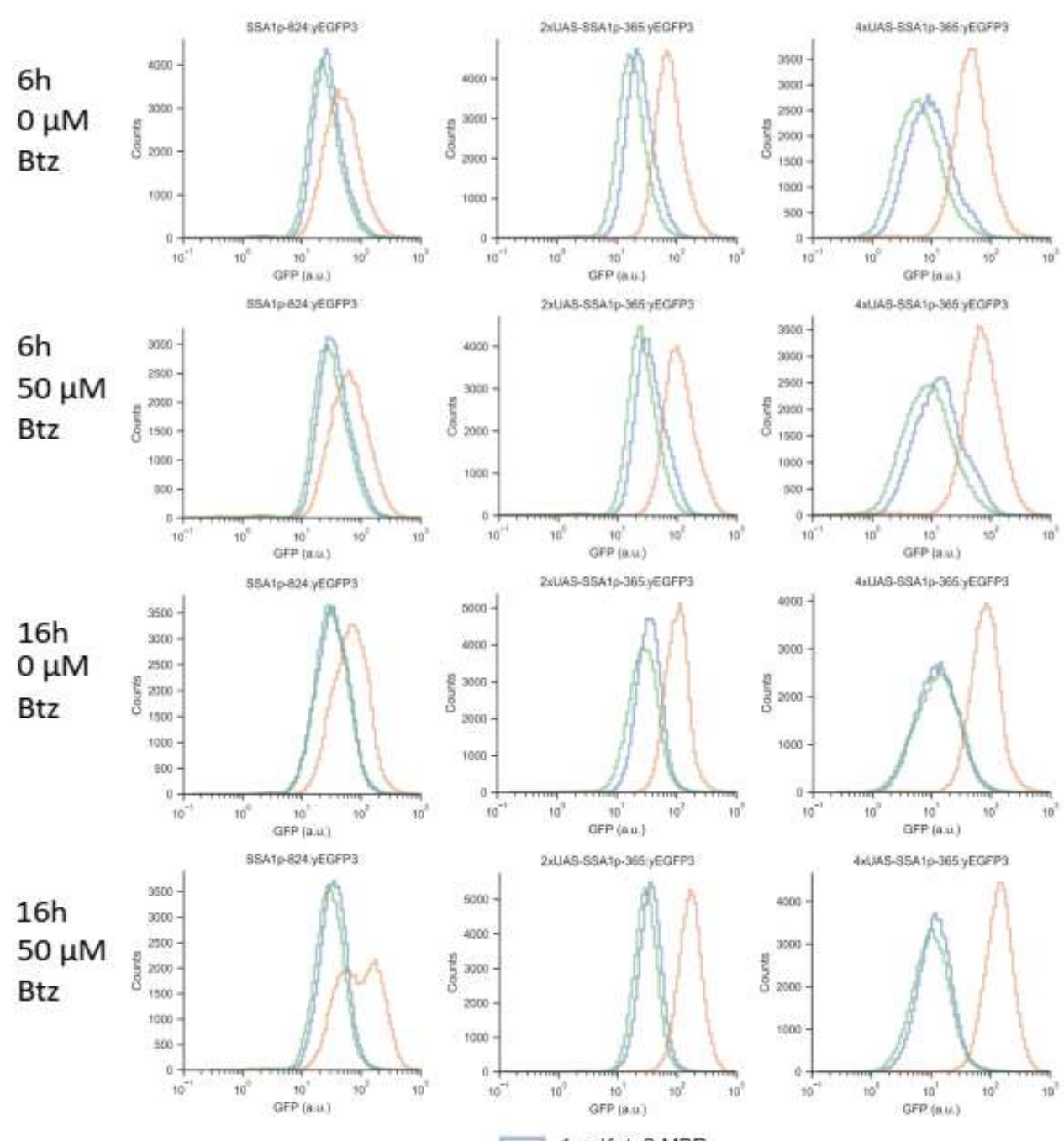

1. mKate2-MBP

2. mKate2-LipPks1

3. Only-reporter 
Supplementary Figure 10. Flow cytometry histograms of 4xUAS-SSA1 reporter output and mKate2 fluorescence in strains expressing soluble (mKate2-MBP) and insoluble (mKate2-LipPks1) protein baits from different promoters. Fluorescence distributions correspond to all events recorded for Figures 4A-B.
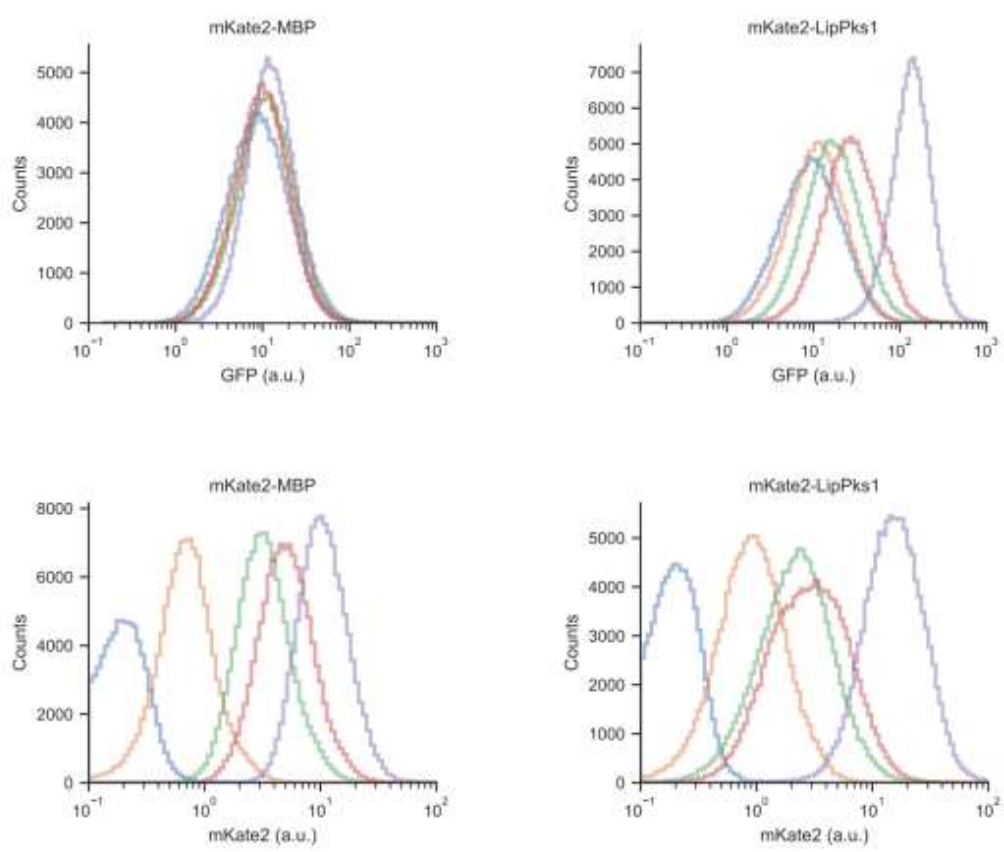

1. REV1p

2. RPL18Bp

3. PGK1p

4. TEF1p

5. $\mathrm{TDH} 3 \mathrm{p}$

Supplementary Figure 11. DMSO toxicity in yeast strains expressing protein baits and 4xUAS-SSA1p-365:yEGFP3 reporter. Growth curves of strains expressing protein baits and control grown at DMSO concentrations $0-10 \%$

A

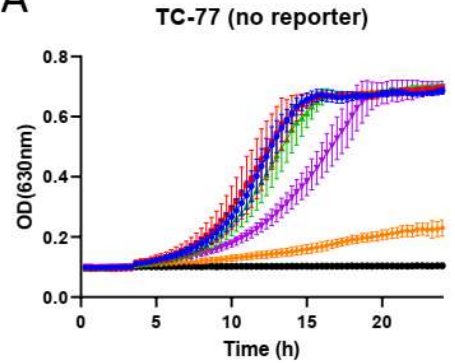

C
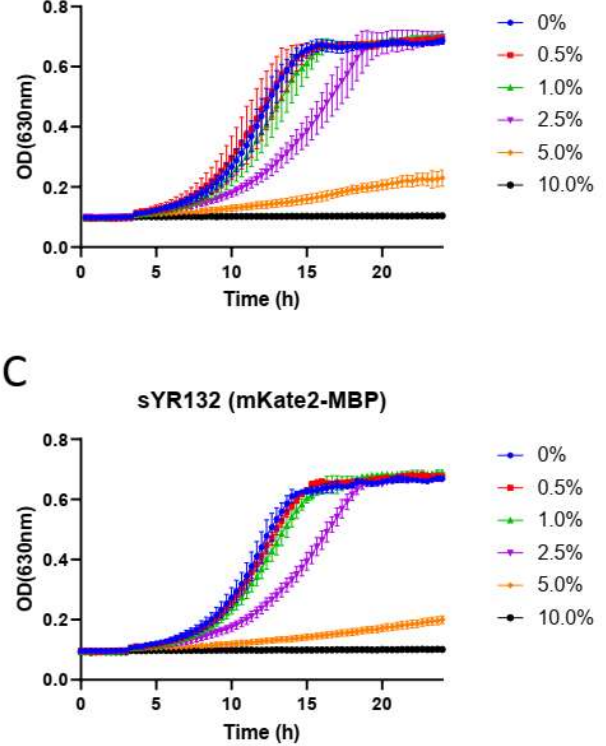

B

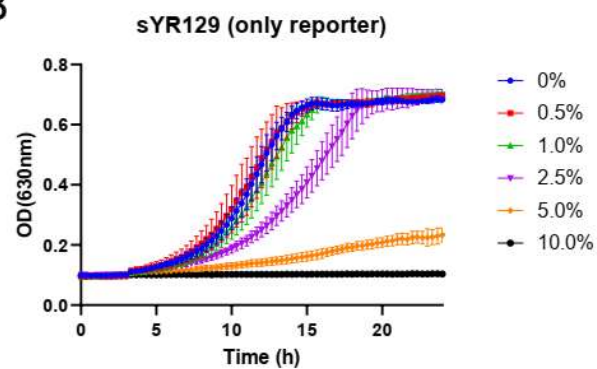

D

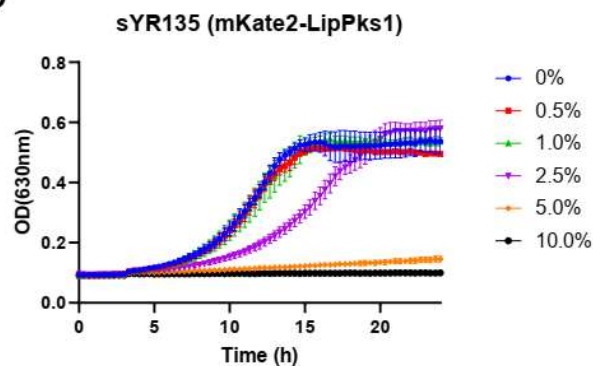


Supplementary Figure 12. Effect of Bortezomib (Btz) in mKate2-MBP and mKateLipPks1 insoluble fractions. A) Western blot of insoluble fractions of strains expressing mKate2-MBP and mKate2-LipPks1 grown for $16 \mathrm{~h}$ in MM with or without $50 \mu \mathrm{M}$ Bortezomib (Btz). B-C) Quantification of bands of insoluble fractions of Western blots from panel A. $n=3-$ 4. Bars represent the mean, and error bars the standard deviation of all data points. Asterisks represent statistical significance between conditions indicated by horizontal bar ends: $*=$ $\mathrm{p}<0.05$.

A

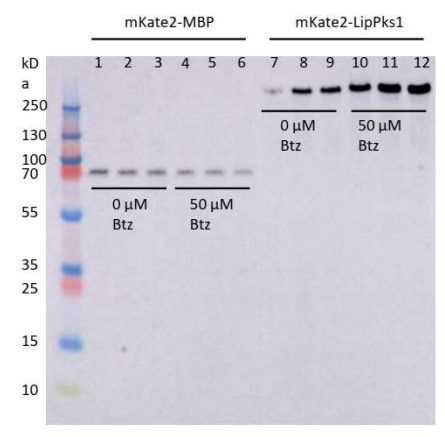

B

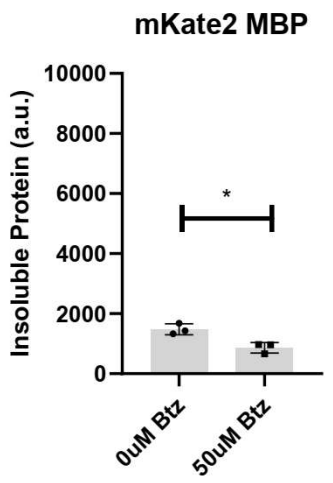

C

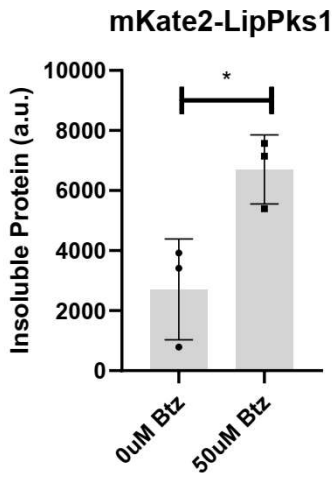

Supplementary Figure 13. 4xUAS-SSA1p-365:yEGFP3 reporter output and mKate2 fluorescence of strains expressing protein baits in cultures supplemented with 1-250 $\mu \mathrm{M}$ Bortezomib (Btz). Fluorescence distributions correspond to all events recorded for Figures 4C-D.
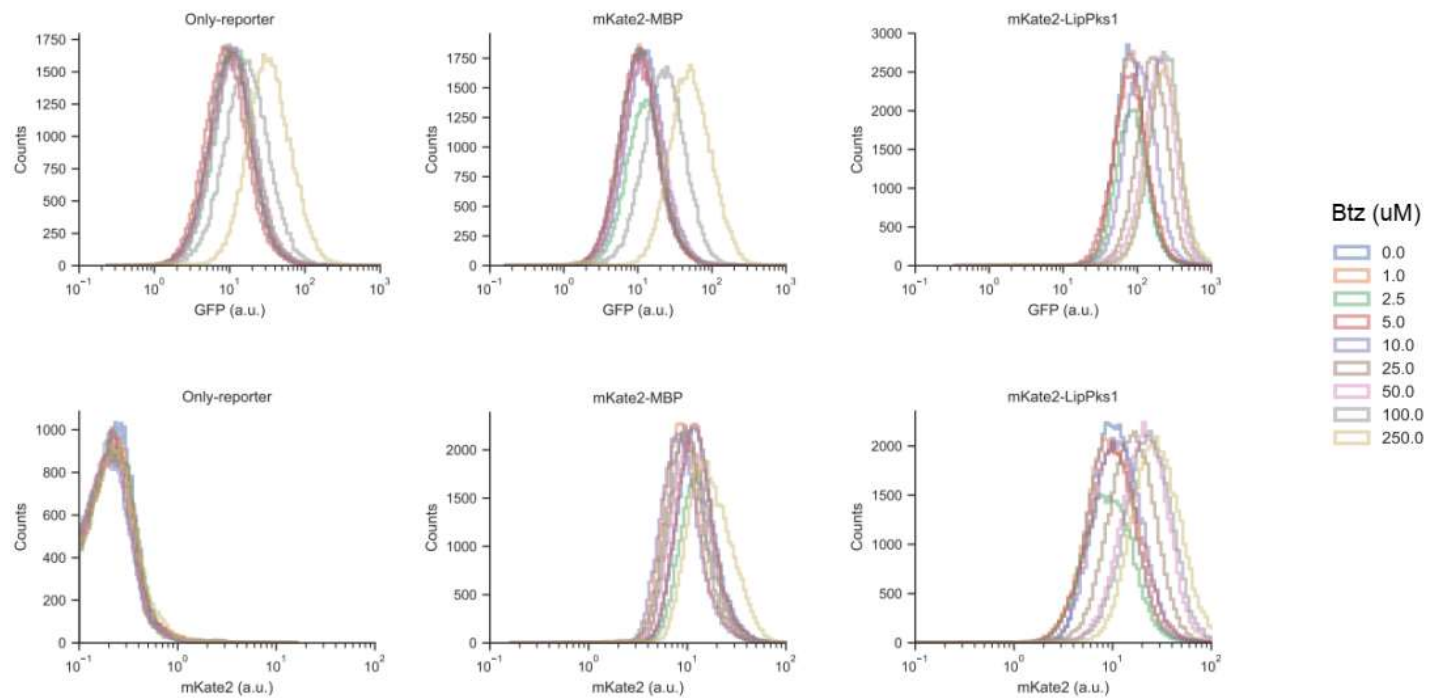
Supplementary Figure 14. Effect of Bortezomib at different concentrations on mKate2LipPks1 insoluble fraction. A) Western blot of insoluble fractions of a strain expressing mKate2-LipPks1 grown for $16 \mathrm{~h}$ in MM with 0-100 $\mu \mathrm{M}$ Bortezomib (Btz). B) Correlation between insoluble protein fractions of mKate2-LipPks1 in A (x-axis) and GFP values of the same cultures (y-axis). $n=3$. Bars represent the mean, and error bars the standard deviation of all data points.

A

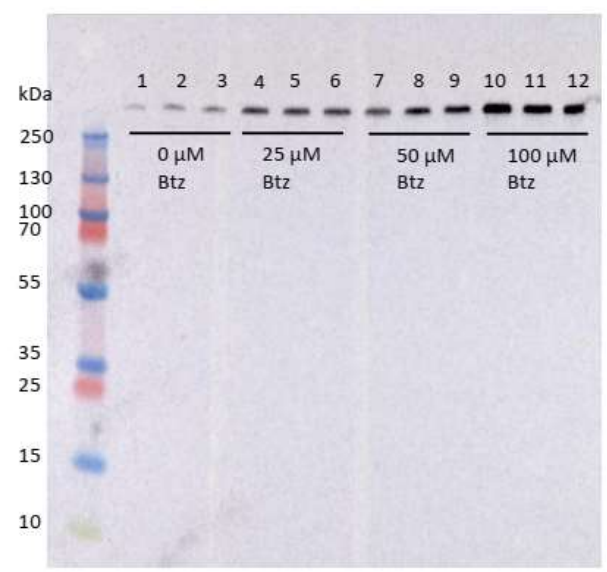

B

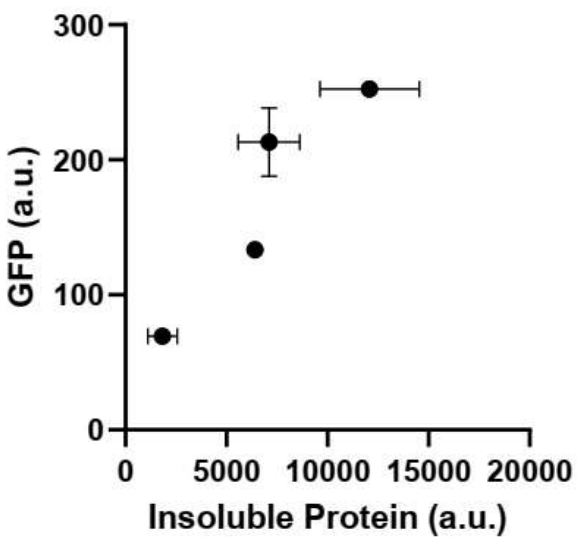

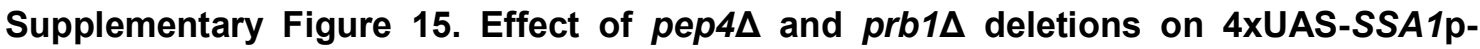
365:yEGFP3 reporter performance. Yeast strains harboring reporter constructs were grown for $6 \mathrm{~h}$ or $16 \mathrm{~h}$ and assessed by flow cytometry ( $\mathrm{n}=6 \times 10,000$ events). A) GFP fold-change of strains. B) Absolute GFP reporter output of strains. a.u. = arbitrary units. For each strain, fold-change was calculated by normalizing GFP geometric means for each replicate $(10,000$ events) to the GFP geometric mean of all mKate2-MBP replicates (40,000 or 60,000 events). Bars represent the mean and error bars SD of data points. Asterisks represent statistical significance between conditions indicated by horizontal bar ends: $* * * *=p<0.0001,{ }^{* * *}=$ $p<0.001,{ }^{* *}=p<0.001, *=p<0.05, n s=$ not significant $(p>0.05)$.

A

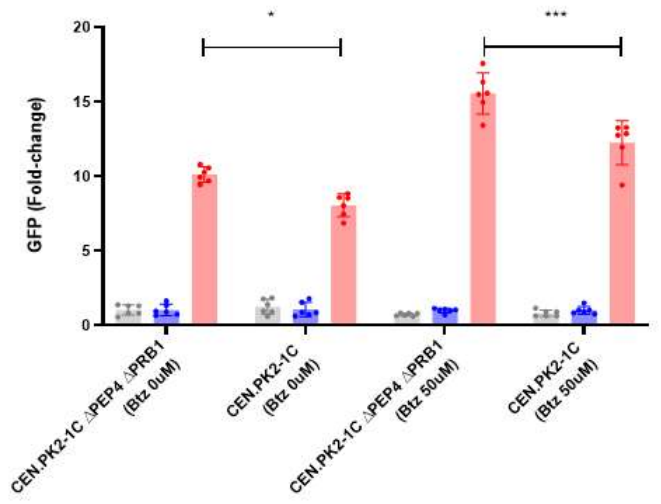

B

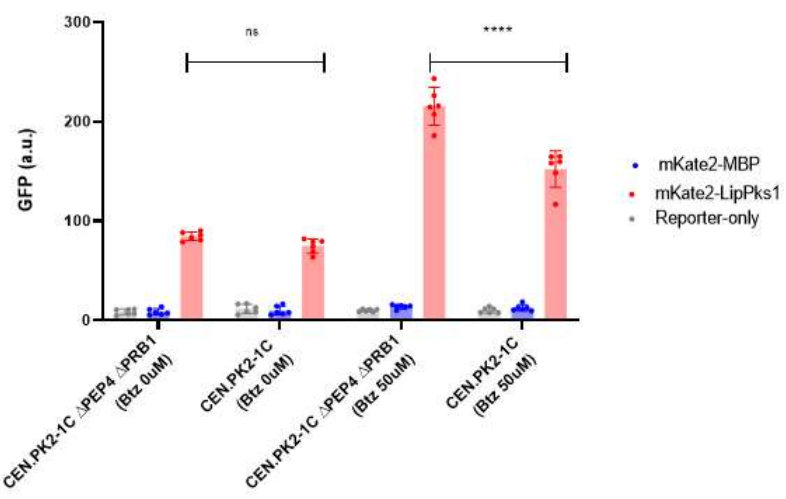


Supplementary Figure 16. yEGFP3 is expressed as a soluble protein in yeast cytosol. Microscopy image of yeast strain expressing yEGFP3.

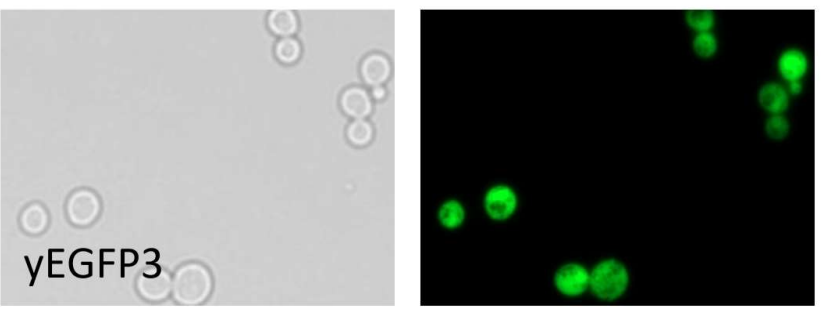

Supplementary Table 1. Plasmids used in this study

\begin{tabular}{|c|c|c|c|}
\hline Plasmid & Expression cassette & $\begin{array}{l}\text { Antibiotic } \\
\text { for } \\
\text { selection }\end{array}$ & Reference \\
\hline pRS414-TEF1p-Cas9-CYC1t (pCas9) & TEF1p-Cas9-CYC1t & Ampicillin & 1 \\
\hline pcfb6909 & $2 \mu$, pESC-LEU2-SNR52p-XII-5_gRNA-SUP4t & Ampicillin & 2 \\
\hline pcfb6901 & $2 \mu$, pESC-LEU2-SNR52p-X-4_gRNA-SUP4t & Ampicillin & This work \\
\hline pDRS90 & XII-5: TDH3p:mKate2-IDP1t & Ampicillin & This work \\
\hline pYR11 & XII-5:TDH3p:his6-mKate2-MBP-IDP1t & Ampicillin & This work \\
\hline pYR14 & XII-5:TDH3p:his6-mKate2-LipPks1-IDP1t & Ampicillin & This work \\
\hline pYR19 & $\mathrm{X}-4: A H A 1 \mathrm{p}-382: \mathrm{uGFP}-\mathrm{CYC} 1 \mathrm{t}$ & Ampicillin & This work \\
\hline pYR21 & $\mathrm{X}-4:$ :PR6p-254:uGFP-CYC1t & Ampicillin & This work \\
\hline pYR25 & $\mathrm{X}-4: H S C 82 \mathrm{p}-384: \mathrm{uGFP}-\mathrm{CYC} 1 \mathrm{t}$ & Ampicillin & This work \\
\hline pYR26 & $\mathrm{X}-4: H S P 42 \mathrm{p}_{-1000}: \mathrm{uGFP}-\mathrm{CYC} 1 \mathrm{t}$ & Ampicillin & This work \\
\hline pYR43 & $\mathrm{X}-4: H S P 78 \mathrm{p}-387: \mathrm{uGFP}-\mathrm{CYC} 1 \mathrm{t}$ & Ampicillin & This work \\
\hline pYR28 & $\mathrm{X}-4: H S P 82 \mathrm{p}_{-858}: \mathrm{uGFP}-\mathrm{CYC} 1 \mathrm{t}$ & Ampicillin & This work \\
\hline pYR29 & $\mathrm{X}-4: H S P 104 \mathrm{p}_{-467}: \mathrm{uGFP}-\mathrm{CYC1t}$ & Ampicillin & This work \\
\hline pYR33 & $X-4: S I S 1 p_{-501: \text { UGFP-CYC1t }}$ & Ampicillin & This work \\
\hline pYR34 & $\mathrm{X}-4: S S A 1 p_{-824: U G F P-C Y C 1 t}$ & Ampicillin & This work \\
\hline pYR36 & X-4:SSA2P-511:uGFP-CYC1t & Ampicillin & This work \\
\hline pYR38 & 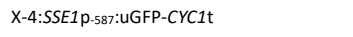 & Ampicillin & This work \\
\hline pYR39 & 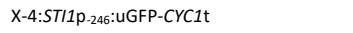 & Ampicillin & This work \\
\hline pYR61 & $\mathrm{X}-4: 1 \mathrm{xHSE}-$ GAL1p-231:uGFP-CYC1t & Ampicillin & This work \\
\hline pYR62 & $\mathrm{X}-4: 2 \mathrm{xHSE}-$ GAL1p-231:uGFP-CYC1t & Ampicillin & This work \\
\hline pYR63 & $\mathrm{X}-4: 4 \mathrm{xHSE}-$ GAL1p-231:uGFP-CYC1t & Ampicillin & This work \\
\hline pYR64 & $\mathrm{X}-4: 8 \mathrm{xHSE}-$ GAL1p-231:uGFP-CYC1t & Ampicillin & This work \\
\hline pYR48 & $\mathrm{X}-4: 1 \mathrm{xUAS}-H S P 82 \mathrm{p}-324: \mathrm{uGFP}-\mathrm{CYC} 1 \mathrm{t}$ & Ampicillin & This work \\
\hline pYR54 & $\mathrm{X}-4: 1 \mathrm{xUAS}-S I S 1 p_{-345}$ :uGFP-CYC1t & Ampicillin & This work \\
\hline pYR52 & $\mathrm{X}-4: 1 \mathrm{xUAS}-\mathrm{SSA} 1 \mathrm{p}_{-365}$ :uGFP-CYC1t & Ampicillin & This work \\
\hline pYR55 & X-4:2xUAS-HSP82p-324:UGFP-CYC1t & Ampicillin & This work \\
\hline pYR56 & $\mathrm{X}-4: 4 \mathrm{xUAS}-H S P 82 \mathrm{p}-324: \mathrm{uGFP}-\mathrm{CYC} 1 \mathrm{t}$ & Ampicillin & This work \\
\hline pYR57 & 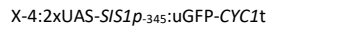 & Ampicillin & This work \\
\hline pYR58 & $\mathrm{X}-4: 4 \mathrm{xUAS}-S I S 1 p_{-345}:$ uGFP-CYC1t & Ampicillin & This work \\
\hline pYR59 & $\mathrm{X}-4: 2 \mathrm{x}$ UAS-SSA1 $\mathrm{p}_{-365}:$ uGFP-CYC1t & Ampicillin & This work \\
\hline
\end{tabular}




\begin{tabular}{|c|c|c|c|}
\hline pYR60 & $\mathrm{X}-4: 4 \mathrm{xUAS}-\mathrm{SSA} 1 \mathrm{p}-365: \mathrm{uGFP}-\mathrm{CYC} 1 \mathrm{t}$ & Ampicillin & This work \\
\hline pYR45 & $\mathrm{X}-4: H S P 82 \mathrm{p}-858$ :yEGFP3-CYC1t & Ampicillin & This work \\
\hline pYR46 & $\mathrm{X}-4: S / S 1$-501:yEGFP3-CYC1t & Ampicillin & This work \\
\hline pYR47 & $\mathrm{X}-4: S S A 1 \mathrm{p}_{-824: y E G F P 3-C Y C 1 \mathrm{t}}$ & Ampicillin & This work \\
\hline pDRS86 & $\mathrm{X}-4: 2 \mathrm{xUAS}-H S P 82 \mathrm{p}-324 \mathrm{yEEFP3}-\mathrm{CYC1 \textrm {t }}$ & Ampicillin & This work \\
\hline pYR65 & $\mathrm{X}-4: 4 \mathrm{xUAS}-H S P 82 \mathrm{p}-324: \mathrm{yEGFP3-CYC1 \textrm {t }}$ & Ampicillin & This work \\
\hline pYR67 & $\mathrm{X}-4: 2 \mathrm{x} U A \mathrm{AS}-\mathrm{SSA} 1 \mathrm{p}_{-365} \mathrm{yEGFP3-CYC1 \textrm {t }}$ & Ampicillin & This work \\
\hline pYR69 & $\mathrm{X}-4: 4 \mathrm{x}$ UAS-SSA1p-365:yEGFP3-CYC1t & Ampicillin & This work \\
\hline pDRS61 & XII-5: RPL18Bp:his6-mKate2-MBP-IDP1t & Ampicillin & This work \\
\hline pDRS63 & XII-5: REV1p:his6-mKate2-MBP-IDP1t & Ampicillin & This work \\
\hline pYR70 & $\mathrm{X}-4: 4 \mathrm{xUAS}-\mathrm{SSA} 1 \mathrm{p}-365: \mathrm{mKate} 2-\mathrm{CYC} 1 \mathrm{t}$ & Ampicillin & This work \\
\hline
\end{tabular}

Supplementary Table 2. In vivo assembled cassettes used in this study

\begin{tabular}{|l|l|l|}
\hline In vivo-assembled expression cassette & Cassette & Reference \\
\hline aDRS349 & XII-5:TEF1p:his6-mKate2-MBP & This work \\
\hline aDRS346 & XII-5:PGK1p:his6-mKate2-MBP & This work \\
\hline aDRS347 & XII-5:RNR2p:his6-mKate2-MBP & This work \\
\hline aDRS348 & XII-5:TEF1p:his6-mKate2-LipPks1 & This work \\
\hline aDRS342 & XII-5:PGK1p:his6-mKate2-LipPks1 & This work \\
\hline aDRS343 & XII-5:RPL18Bp:his6-mKate2-LipPks1 & This work \\
\hline aDRS344 & XII-5:RNR2p:his6-mKate2-LipPks1 & This work \\
\hline aDRS345 & XII-5:REV1p:his6-mKate2-LipPks1 & This work \\
\hline aDRS354 & XII-5:TDH3p:his6-mKate2-Sup35 & This work \\
\hline aDRS355 & XII-5:TDH3p:his6-mKate2-Rnq1 & This work \\
\hline aDRS357 & XII-5:TDH3p:his6-mKate2-Cyc8 & This work \\
\hline aDRS247 & XII-5:TDH3p:his6-YFPm4-SpHIS5 & This work \\
\hline aDRS318 & XII-5:TDH3p:his6-yEGFP & This work \\
\hline aDRS371 & HSP104: GFP-His5 & This work \\
\hline
\end{tabular}

\section{Supplementary Table 3. Yeast strains used in this study}

\begin{tabular}{|c|c|c|c|c|c|c|c|}
\hline Strain & Use & $\begin{array}{l}\text { Parental } \\
\text { strain }\end{array}$ & $\begin{array}{l}\text { Yeast } \\
\text { Integrative } \\
\text { Plasmid }\end{array}$ & $\begin{array}{l}\text { In vivo- } \\
\text { assembled } \\
\text { expression } \\
\text { cassette }\end{array}$ & $\begin{array}{l}\text { Episomal/centromeric } \\
\text { plasmid }\end{array}$ & Genotype & Reference \\
\hline $\begin{array}{l}\text { CEN.PK2- } \\
1 C\end{array}$ & $\begin{array}{l}\text { Parental } \\
\text { strain }\end{array}$ & - & - & - & - & $\begin{array}{l}\text { MATa ura3-52 trp1-289 leu2-3,112 his3 } \triangle 1 \text { MAL2-8C } \\
\text { SUC2 }\end{array}$ & Peter Kötter \\
\hline sDRS23 & $\begin{array}{l}\text { Parental } \\
\text { strain }\end{array}$ & CEN.PK2-1C & - & - & pCas9 & $\begin{array}{l}\text { MATa ura3-52 trp1-289 leu2-3,112 his3 } 11 \text { MAL2-8C } \\
\text { SUC2 Cas9 }\end{array}$ & This work \\
\hline TC-77 & $\begin{array}{l}\text { Parental } \\
\text { strain }\end{array}$ & CEN.PK2-1C & - & - & pCas9 & $\begin{array}{l}\text { MATa ura3-52 trp1-289 leu2-3,112 his3 } \triangle 1 \text { MAL2-8C } \\
\text { SUC2 } \triangle \text { PEP4 } \triangle \text { PRB1 pCas9 }\end{array}$ & This work \\
\hline sYR10 & $\begin{array}{l}\text { Parental } \\
\text { strain }\end{array}$ & TC-77 & pYR11 & - & pCas9 & $\begin{array}{l}\text { MATa ura3-52 trp1-289 leu2-3,112 his3 } 11 \text { MAL2-8C } \\
\text { SUC2 } \triangle \text { PEP4 } \triangle \text { PRB1 pCas9 TDH3p:his6-mKate2-MBP }\end{array}$ & This work \\
\hline sYR11 & $\begin{array}{l}\text { Parental } \\
\text { strain }\end{array}$ & TC-77 & pYR14 & - & pCas9 & $\begin{array}{l}\text { MATa ura3-52 trp1-289 leu2-3,112 his3 } \triangle 1 \text { MAL2-8C } \\
\text { SUC2 } \triangle \text { PEP4 } \triangle \text { PRB1 pCas9 TDH3p:his6-mKate2- } \\
\text { LipPks1 }\end{array}$ & This work \\
\hline sYR06 & $\begin{array}{l}\text { F1A, F1B, } \\
\text { F1C, F1D, } \\
\text { F1E }\end{array}$ & TC-77 & - & - & - & $\begin{array}{l}\text { MATa ura3-52 trp1-289 leu2-3,112 his3 } \triangle 1 \text { MAL2-8C } \\
\text { SUC2 } \triangle \text { PEP4 } \triangle \text { PRB1 }\end{array}$ & This work \\
\hline sYR07 & $\begin{array}{l}\text { F1A, F1B, } \\
\text { F1C, F1D, } \\
\text { F1E }\end{array}$ & TC-77 & pDRS90 & - & - & $\begin{array}{l}\text { MATa ura3-52 trp1-289 leu2-3,112 his3 } \triangle 1 \text { MAL2-8C } \\
\text { SUC2 } \triangle \text { PEP4 } \triangle \text { PRB1 TDH3p:mKate2 }\end{array}$ & This work \\
\hline sYR08 & $\begin{array}{l}\text { F1A, F1B, } \\
\text { F1C, F1D, } \\
\text { F1E }\end{array}$ & TC-77 & pYR11 & - & - & $\begin{array}{l}\text { MATa ura3-52 trp1-289 leu2-3,112 his3 } \triangle 1 \text { MAL2-8C } \\
\text { SUC2 } \triangle \text { PEP4 } \triangle \text { PRB1 TDH3p:his6-mKate2-MBP }\end{array}$ & This work \\
\hline sYR09 & $\begin{array}{l}\text { F1A, F1B, } \\
\text { F1C, F1D, } \\
\text { F1E }\end{array}$ & TC-77 & pYR14 & - & - & $\begin{array}{l}\text { MATa ura3-52 trp1-289 leu2-3,112 his3 } \triangle 1 \text { MAL2-8C } \\
\text { SUC2 } \triangle \text { PEP4 } \triangle \text { PRB1 TDH3p:his6-mKate2-LipPks1 }\end{array}$ & This work \\
\hline sYR64 & F2B & TC-77 & pYR19 & - & pCas9 & $\begin{array}{l}\text { MATa ura3-52 trp1-289 leu2-3,112 his3 } 31 \text { MAL2-8C } \\
\text { SUC2 } \triangle \text { PEP4 } \triangle \text { PRB1 pCas9 AHA1p-382:UGFP }\end{array}$ & This work \\
\hline sYR66 & F2B & TC-77 & pYR21 & - & pCas9 & $\begin{array}{l}\text { MATa ura3-52 trp1-289 leu2-3,112 his3 } 11 \text { MAL2-8C } \\
\text { SUC2 } \triangle \text { PEP4 } \triangle \text { PRB1 pCas9 CPR6p-254:uGFP }\end{array}$ & This work \\
\hline
\end{tabular}




\begin{tabular}{|c|c|c|c|c|c|c|c|}
\hline sYR67 & F2B & TC-77 & pYR25 & - & pCas9 & $\begin{array}{l}\text { MATa ura3-52 trp1-289 leu2-3,112 his3 } 11 \text { MAL2-8C } \\
\text { SUC2 } \triangle \text { PEP4 } \triangle \text { PRB1 pCas9 HSC82p-384:UGFP }\end{array}$ & This work \\
\hline sYR28 & F2B & TC-77 & pYR26 & - & pCas9 & $\begin{array}{l}\text { MATa ura3-52 trp1-289 leu2-3,112 his3 } 11 \text { MAL2-8C } \\
\text { SUC2 } \triangle \text { PEP4 } \triangle \text { PRB1 pCas9 HSP42p-1000:uGFP }\end{array}$ & This work \\
\hline sYR74 & F2B & TC-77 & pYR43 & - & pCas9 & $\begin{array}{l}\text { MATa ura3-52 trp1-289 leu2-3,112 his3 } \triangle 1 \text { MAL2-8C } \\
\text { SUC2 } \triangle \text { PEP4 } \triangle \text { PRB1 pCas9 HSP78p-387:uGFP }\end{array}$ & This work \\
\hline sYR29 & $\mathrm{F} 2 \mathrm{~B}, \mathrm{~F} 3 \mathrm{~A}$ & TC-77 & pYR28 & - & pCas9 & $\begin{array}{l}\text { MATa ura3-52 trp1-289 leu2-3,112 his3 } \Delta 1 \text { MAL2-8C } \\
\text { SUC2 } \triangle \text { PEP4 } \triangle \text { PRB1 pCas9 HSP82p-858:UGFP }\end{array}$ & This work \\
\hline sYR30 & F2B & TC-77 & pYR29 & - & pCas9 & $\begin{array}{l}\text { MATa ura3-52 trp1-289 leu2-3,112 his3 } \triangle 1 \text { MAL2-8C } \\
\text { SUC2 } \triangle \text { PEP4 } \triangle \text { PRB1 pCas9 HSP104p-467:UGFP }\end{array}$ & This work \\
\hline sYR31 & F2B, F3A & TC-77 & pYR33 & - & pCas9 & $\begin{array}{l}\text { MATa ura3-52 trp1-289 leu2-3,112 his3 } \Delta 1 \text { MAL2-8C } \\
\text { SUC2 } \triangle \text { PEP4 } \triangle \text { PRB1 pCas9 SIS1p-501:uGFP }\end{array}$ & This work \\
\hline sYR32 & F2B, F3A & TC-77 & pYR34 & - & pCas9 & $\begin{array}{l}\text { MATa ura3-52 trp1-289 leu2-3,112 his3 } \Delta 1 \text { MAL2-8C } \\
\text { SUC2 } \triangle \text { PEP4 } \triangle \text { PRB1 pCas9 SSA1p-824:UGFP }\end{array}$ & This work \\
\hline sYR71 & F2B & TC-77 & pYR36 & - & pCas9 & $\begin{array}{l}\text { MATa ura3-52 trp1-289 leu2-3,112 his3 } \triangle 1 \text { MAL2-8C } \\
\text { SUC2 } \triangle \text { PEP4 } \triangle \text { PRB1 pCas9 SSA2p-511:UGFP }\end{array}$ & This work \\
\hline sYR73 & F2B & TC-77 & pYR38 & - & pCas9 & $\begin{array}{l}\text { MATa ura3-52 trp1-289 leu2-3,112 his3 } \triangle 1 \text { MAL2-8C } \\
\text { SUC2 } \triangle \text { PEP4 } \triangle \text { PRB1 pCas9 SSE1p-587:UGFP }\end{array}$ & This work \\
\hline sYR33 & F2B & TC-77 & pYR39 & - & pCas9 & $\begin{array}{l}\text { MATa ura3-52 trp1-289 leu2-3,112 his3 } \Delta 1 \text { MAL2-8C } \\
\text { SUC2 } \triangle \text { PEP4 } \triangle \text { PRB1 pCas9 ST/1p-246:uGFP }\end{array}$ & This work \\
\hline sYR95 & F2B & TC-77 & pYR61 & - & pCas9 & $\begin{array}{l}\text { MATa ura3-52 trp1-289 leu2-3,112 his3 } 11 \text { MAL2-8C } \\
\text { SUC2 } \triangle \text { PEP4 } \triangle \text { PRB1 pCas9 1xHSE-GAL1p-231:UGFP }\end{array}$ & This work \\
\hline sYR96 & F2B & TC-77 & pYR62 & - & pCas9 & $\begin{array}{l}\text { MATa ura3-52 trp1-289 leu2-3,112 his3 } \triangle 1 \text { MAL2-8C } \\
\text { SUC2 } \triangle \text { PEP4 } \triangle \text { PRB1 pCas9 2xHSE-GAL1p-231:UGFP }\end{array}$ & This work \\
\hline sYR97 & F2B & TC-77 & pYR63 & - & pCas9 & $\begin{array}{l}\text { MATa ura3-52 trp1-289 leu2-3,112 his3 } \triangle 1 \text { MAL2-8C } \\
\text { SUC2 } \triangle \text { PEP4 } \triangle \text { PRB1 pCas9 4xHSE-GAL1p-231:UGFP }\end{array}$ & This work \\
\hline sYR98 & F2B & TC-77 & pYR64 & - & pCas9 & $\begin{array}{l}\text { MATa ura3-52 trp1-289 leu2-3,112 his3 } \triangle 1 \text { MAL2-8C } \\
\text { SUC2 } \triangle \text { PEP4 } \triangle \text { PRB1 pCas9 8xHSE-GAL1p-231:uGFP }\end{array}$ & This work \\
\hline sYR40 & F2B & sYR10 & pYR19 & - & pCas9 & $\begin{array}{l}\text { MATa ura3-52 trp1-289 leu2-3,112 his3 } 1 \text { MAL2-8C } \\
\text { SUC2 } \triangle \text { PEP4 } \triangle \text { PRB1 pCas9 TDH3p:his6-mKate2-MBP } \\
\text { AHA1p-382:UGFP }\end{array}$ & This work \\
\hline sYR42 & F2B & sYR10 & pYR21 & - & pCas9 & $\begin{array}{l}\text { MATa ura3-52 trp1-289 leu2-3,112 his3 } 1 \text { MAL2-8C } \\
\text { SUC2 } \triangle \text { PEP4 } \triangle \text { PRB1 pCas9 TDH3p:his6-mKate2-MBP } \\
\text { CPR6p-254:UGFP }\end{array}$ & This work \\
\hline $\begin{array}{l}\text { sYR43 } \\
\end{array}$ & F2B & sYR10 & pYR25 & - & pCas9 & $\begin{array}{l}\text { MATa ura3-52 trp1-289 leu2-3,112 his3 } 1 \text { MAL2-8C } \\
\text { SUC2 } \triangle \text { PEP4 } \triangle \text { PRB1 pCas9 TDH3p:his6-mKate2-MBP } \\
\text { HSC82p-384:UGFP }\end{array}$ & This work \\
\hline sYR12 & F2B & sYR10 & pYR26 & - & pCas9 & $\begin{array}{l}\text { MATa ura3-52 trp1-289 leu2-3,112 his3 } 1 \text { MAL2-8C } \\
\text { SUC2 } \triangle \text { PEP4 } \triangle \text { PRB1 pCas9 TDH3p:his6-mKate2-MBP } \\
\text { HSP42p-1000:UGFP }\end{array}$ & This work \\
\hline sYR50 & F2B & sYR10 & pYR43 & - & pCas9 & $\begin{array}{l}\text { MATa ura3-52 trp1-289 leu2-3,112 his3 } 1 \text { MAL2-8C } \\
\text { SUC2 } \triangle \text { PEP4 } \triangle \text { PRB1 pCas9 TDH3p:his6-mKate2-MBP } \\
\text { HSP78p-387:UGFP }\end{array}$ & This work \\
\hline sYR13 & F2B, F3A & sYR10 & pYR28 & - & pCas9 & $\begin{array}{l}\text { MATa ura3-52 trp1-289 leu2-3,112 his3 } 1 \text { MAL2-8C } \\
\text { SUC2 } \triangle \text { PEP4 } \triangle \text { PRB1 pCas9 TDH3p:his6-mKate2-MBP } \\
\text { HSP82p-858:UGFP }\end{array}$ & This work \\
\hline sYR14 & F2B & sYR10 & pYR29 & - & pCas9 & $\begin{array}{l}\text { MATa ura3-52 trp1-289 leu2-3,112 his3 } 1 \text { MAL2-8C } \\
\text { SUC2 } \triangle \text { PEP4 } \triangle \text { PRB1 pCas9 TDH3p:his6-mKate2-MBP } \\
\text { HSP104p-467:UGFP }\end{array}$ & This work \\
\hline sYR15 & $F 2 B, F 3 A$ & sYR10 & pYR33 & - & pCas9 & $\begin{array}{l}\text { MATa ura3-52 trp1-289 leu2-3,112 his3 } \triangle 1 \text { MAL2-8C } \\
\text { SUC2 } \triangle \text { PEP4 } \triangle \text { PRB1 pCas9 TDH3p:his6-mKate2-MBP } \\
\text { SIS1p-501:UGFP }\end{array}$ & This work \\
\hline sYR16 & $F 2 B, F 3 A$ & sYR10 & pYR34 & - & pCas9 & $\begin{array}{l}\text { MATa ura3-52 trp1-289 leu2-3,112 his3 } \Delta 1 \text { MAL2-8C } \\
\text { SUC2 } \triangle \text { PEP4 } \triangle \text { PRB1 pCas9 TDH3p:his6-mKate2-MBP } \\
\text { SSA1p-824:UGFP }\end{array}$ & This work \\
\hline sYR47 & F2B & $\begin{array}{l}\text { sYR10 } \\
\end{array}$ & pYR36 & - & pCas9 & $\begin{array}{l}\text { MATa ura3-52 trp1-289 leu2-3,112 his3 } 1 \text { MAL2-8C } \\
\text { SUC2 } \triangle \text { PEP4 } \triangle \text { PRB1 pCas9 TDH3p:his6-mKate2-MBP } \\
\text { SSA2p-511:uGFP }\end{array}$ & This work \\
\hline sYR49 & F2B & sYR10 & $\begin{array}{l}\text { pYR38 } \\
\end{array}$ & - & pCas9 & $\begin{array}{l}\text { MATa ura3-52 trp1-289 leu2-3,112 his3 } 1 \text { MAL2-8C } \\
\text { SUC2 } \triangle \text { PEP4 } \triangle \text { PRB1 pCas9 TDH3p:his6-mKate2-MBP } \\
\text { SSE1p-587:UGFP }\end{array}$ & This work \\
\hline sYR17 & F2B & sYR10 & pYR39 & - & pCas9 & $\begin{array}{l}\text { MATa ura3-52 trp1-289 leu2-3,112 his3 } 1 \text { MAL2-8C } \\
\text { SUC2 } \triangle \text { PEP4 } \triangle \text { PRB1 pCas9 TDH3p:his6-mKate2-MBP } \\
\text { STI1p-246:UGFP }\end{array}$ & This work \\
\hline SYR109 & F2B & sYR10 & pYR61 & - & pCas9 & $\begin{array}{l}\text { MATa ura3-52 trp1-289 leu2-3,112 his3 } 1 \text { MAL2-8C } \\
\text { SUC2 } \triangle \text { PEP4 } \triangle \text { PRB1 pCas9 TDH3p:his6-mKate2-MBP } \\
\text { 1xHSE-GAL1p-231:uGFP }\end{array}$ & This work \\
\hline sYR110 & F2B & sYR10 & pYR62 & - & pCas9 & $\begin{array}{l}\text { MATa ura3-52 trp1-289 leu2-3,112 his3 } 1 \text { MAL2-8C } \\
\text { SUC2 } \triangle \text { PEP4 } \triangle \text { PRB1 pCas9 TDH3p:his6-mKate2-MBP } \\
\text { 2xHSE-GAL1p-231:uGFP }\end{array}$ & This work \\
\hline sYR111 & F2B & sYR10 & pYR63 & - & pCas9 & $\begin{array}{l}\text { MATa ura3-52 trp1-289 leu2-3,112 his3 } 1 \text { MAL2-8C } \\
\text { SUC2 } \triangle \text { PEP4 } \triangle \text { PRB1 pCas9 TDH3p:his6-mKate2-MBP } \\
\text { 4xHSE-GAL1p-231:uGFP }\end{array}$ & This work \\
\hline SYR112 & F2B & $\begin{array}{l}\text { sYR10 } \\
\end{array}$ & $\begin{array}{l}\text { pYR64 } \\
\text {. }\end{array}$ & - & pCas9 & $\begin{array}{l}\text { MATa ura3-52 trp1-289 leu2-3,112 his3 } 1 \text { MAL2-8C } \\
\text { SUC2 } \triangle \text { PEP4 } \triangle \text { PRB1 pCas9 TDH3p:his6-mKate2-MBP } \\
\text { 8xHSE-GAL1p-231:uGFP }\end{array}$ & This work \\
\hline sYR52 & F2B & sYR11 & pYR19 & - & pCas9 & $\begin{array}{l}\text { MATa ura3-52 trp1-289 leu2-3,112 his3 } 11 \text { MAL2-8C } \\
\text { SUC2 } \triangle \text { PEP4 } \triangle \text { PRB1 pCas9 TDH3p:his6-mKate2- } \\
\text { LipPks1 AHA1p-382:uGFP }\end{array}$ & This work \\
\hline sYR54 & $F 2 B$ & sYR11 & $\begin{array}{l}\text { pYR21 } \\
\end{array}$ & - & pCas9 & $\begin{array}{l}\text { MATa ura3-52 trp1-289 leu2-3,112 his3 } 1 \text { MAL2-8C } \\
\text { SUC2 } \triangle \text { PEP4 } \triangle \text { PRB1 pCas9 TDH3p:his6-mKate2- } \\
\text { LipPk1 CPR6p-254:UGFP }\end{array}$ & This work \\
\hline sYR55 & F2B & sYR11 & pYR25 & - & pCas9 & $\begin{array}{l}\text { MATa ura3-52 trp1-289 leu2-3,112 his3 } 1 \text { MAL2-8C } \\
\text { SUC2 } \triangle \text { PEP4 } \triangle \text { PRB1 pCas9 TDH3p:his6-mKate2- } \\
\text { LipPks1 HSC82p-384:UGFP }\end{array}$ & This work \\
\hline sYR18 & F2B & sYR11 & pYR26 & - & pCas9 & $\begin{array}{l}\text { MATa ura3-52 trp1-289 leu2-3,112 his3 } 1 \text { MAL2-8C } \\
\text { SUC2 } \triangle \text { PEP4 } \triangle \text { PRB1 pCas9 TDH3p:his6-mKate2- } \\
\text { LipPks1 HSP42p-1000:UGFP }\end{array}$ & This work \\
\hline sYR62 & F2B & sYR11 & pYR43 & - & pCas9 & $\begin{array}{l}\text { MATa ura3-52 trp1-289 leu2-3,112 his3 } 1 \text { MAL2-8C } \\
\text { SUC2 } \triangle \text { PEP4 } \triangle \text { PRB1 pCas9 TDH3p:his6-mKate2- } \\
\text { LipPks1 HSP78p-387:UGFP }\end{array}$ & This work \\
\hline
\end{tabular}




\begin{tabular}{|c|c|c|c|c|c|c|c|}
\hline sYR19 & F2B, F3A & sYR11 & $\begin{array}{l}\text { pYR28 } \\
\end{array}$ & - & pCas9 & $\begin{array}{l}\text { MATa ura3-52 trp1-289 leu2-3,112 his3 } 11 \text { MAL2-8C } \\
\text { SUC2 } \triangle \text { PEP4 } \triangle \text { PRB1 pCas9 TDH3p:his6-mKate2- } \\
\text { LipPks1 HSP82p-858:uGFP }\end{array}$ & This work \\
\hline sYR20 & F2B & sYR11 & pYR29 & - & pCas9 & $\begin{array}{l}\text { MATa ura3-52 trp1-289 leu2-3,112 his3 } \triangle 1 \text { MAL2-8C } \\
\text { SUC2 } \triangle \text { PEP4 } \triangle \text { PRB1 pCas9 TDH3p:his6-mKate2- } \\
\text { LipPks1 HSP104p-467:UGFP }\end{array}$ & This work \\
\hline sYR21 & F2B, F3A & sYR11 & pYR33 & - & pCas9 & $\begin{array}{l}\text { MATa ura3-52 trp1-289 leu2-3,112 his3 } \triangle 1 \text { MAL2-8C } \\
\text { SUC2 } \triangle \text { PEP4 } \triangle \text { PRB1 pCas9 TDH3p:his6-mKate2- } \\
\text { LipPks1 SIS1p-501:uGFP }\end{array}$ & This work \\
\hline sYR22 & F2B, F3A & sYR11 & $\begin{array}{l}\text { pYR34 } \\
\end{array}$ & - & pCas9 & $\begin{array}{l}\text { MATa ura3-52 trp1-289 leu2-3,112 his3 } 11 \text { MAL2-8C } \\
\text { SUC2 } \triangle \text { PEP4 } \triangle \text { PRB1 pCas9 TDH3p:his6-mKate2- } \\
\text { LipPks1 SSA1p-824:UGFP }\end{array}$ & This work \\
\hline sYR59 & $\mathrm{F} 2 \mathrm{~B}$ & sYR11 & pYR36 & - & pCas9 & $\begin{array}{l}\text { MATa ura3-52 trp1-289 leu2-3,112 his3 } 1 \text { 1 MAL2-8C } \\
\text { SUC2 } \triangle \text { PEP4 } \triangle \text { PRB1 pCas9 TDH3p:his6-mKate2- } \\
\text { LipPks1 SSA2p-511:uGFP }\end{array}$ & This work \\
\hline sYR61 & $F 2 B$ & sYR11 & pYR38 & - & pCas9 & $\begin{array}{l}\text { MATa ura3-52 trp1-289 leu2-3,112 his3 } 11 \text { MAL2-8C } \\
\text { SUC2 } \triangle \text { PEP4 } \triangle \text { PRB1 pCas9 TDH3p:his6-mKate2- } \\
\text { LipPks1 SSE1p-587:uGFP }\end{array}$ & This work \\
\hline sYR23 & F2B & sYR11 & pYR39 & - & pCas9 & $\begin{array}{l}\text { MATa ura3-52 trp1-289 leu2-3,112 his3 } 11 \text { MAL2-8C } \\
\text { SUC2 } \triangle \text { PEP4 } \triangle \text { PRB1 pCas9 TDH3p:his6-mKate2- } \\
\text { LipPks1 STI1p-246:UGFP }\end{array}$ & This work \\
\hline SYR123 & F2B & sYR11 & $\begin{array}{l}\text { pYR61 } \\
\end{array}$ & - & pCas9 & $\begin{array}{l}\text { MATa ura3-52 trp1-289 leu2-3,112 his3 } 11 \text { MAL2-8C } \\
\text { SUC2 } \triangle \text { PEP4 } \triangle \text { PRB1 pCas9 TDH3p:his6-mKate2- } \\
\text { LipPks1 1xHSE-GAL1p-231:uGFP }\end{array}$ & This work \\
\hline SYR124 & F2B & sYR11 & pYR62 & - & pCas9 & $\begin{array}{l}\text { MATa ura3-52 trp1-289 leu2-3,112 his3 } 11 \text { MAL2-8C } \\
\text { SUC2 } \triangle \text { PEP4 } \triangle \text { PRB1 pCas9 TDH3p:his6-mKate2- } \\
\text { LipPks1 2xHSE-GAL1p-231:UGFP }\end{array}$ & This work \\
\hline sYR125 & $\mathrm{F} 2 \mathrm{~B}$ & sYR11 & pYR63 & - & pCas9 & $\begin{array}{l}\text { MATa ura3-52 trp1-289 leu2-3,112 his3 } \triangle 1 \text { MAL2-8C } \\
\text { SUC2 } \triangle \text { PEP4 } \triangle \text { PRB1 pCas9 TDH3p:his6-mKate2- } \\
\text { LipPks1 4xHSE-GAL1p-231: UGFP }\end{array}$ & This work \\
\hline sYR126 & $F 2 B$ & sYR11 & $\begin{array}{l}\text { pYR64 } \\
\end{array}$ & - & pCas9 & $\begin{array}{l}\text { MATa ura3-52 trp1-289 leu2-3,112 his3 } 11 \text { MAL2-8C } \\
\text { SUC2 } \triangle \text { PEP4 } \triangle \text { PRB1 pCas9 TDH3p:his6-mKate2- } \\
\text { LipPks1 8xHSE-GAL1p-231:uGFP }\end{array}$ & This work \\
\hline sYR85 & F3A & TC-77 & pYR48 & - & pCas9 & $\begin{array}{l}\text { MATa ura3-52 trp1-289 leu2-3,112 his3 } 11 \text { MAL2-8C } \\
\text { SUC2 } \triangle \text { PEP4 } \triangle \text { PRB1 pCas9 1xUAS-HSP82p-324:uGFP }\end{array}$ & This work \\
\hline sYR87 & F3A & TC-77 & pYR52 & - & pCas9 & $\begin{array}{l}\text { MATa ura3-52 trp1-289 leu2-3,112 his3 } 11 \text { MAL2-8C } \\
\text { SUC2 } \triangle \text { PEP4 } \triangle \text { PRB1 pCas9 1xUAS-SIS1p-345:uGFP }\end{array}$ & This work \\
\hline sYR88 & $\mathrm{F} 3 \mathrm{~A}$ & TC-77 & pYR54 & - & pCas9 & $\begin{array}{l}\text { MATa ura3-52 trp1-289 leu2-3,112 his3 } \triangle 1 \text { MAL2-8C } \\
\text { SUC2 } \triangle \text { PEP4 } \triangle \text { PRB1 pCas9 1xUAS-SSA1p-365:uGFP }\end{array}$ & This work \\
\hline sYR89 & F3A & TC-77 & $\begin{array}{l}\text { pYR55 } \\
\end{array}$ & - & pCas9 & $\begin{array}{l}\text { MATa ura3-52 trp1-289 leu2-3,112 his3 } \triangle 1 \text { MAL2-8C } \\
\text { SUC2 } \triangle \text { PEP4 } \triangle \text { PRB1 pCas9 2xUAS-HSP82p-324:uGFP }\end{array}$ & This work \\
\hline sYR90 & F3A & TC-77 & pYR56 & - & pCas9 & $\begin{array}{l}\text { MATa ura3-52 trp1-289 leu2-3,112 his3 } 11 \text { MAL2-8C } \\
\text { SUC2 } \triangle \text { PEP4 } \triangle \text { PRB1 pCas9 4xUAS-HSP82p-324:uGFP }\end{array}$ & This work \\
\hline sYR91 & $\mathrm{F} 3 \mathrm{~A}$ & TC-77 & pYR57 & - & pCas9 & $\begin{array}{l}\text { MATa ura3-52 trp1-289 leu2-3,112 his3 } 11 \text { MAL2-8C } \\
\text { SUC2 } \triangle \text { PEP4 } \triangle \text { PRB1 pCas9 2xUAS-SIS1p-345:uGFP }\end{array}$ & This work \\
\hline sYR92 & F3A & TC-77 & $\begin{array}{l}\text { pYR58 } \\
\end{array}$ & - & pCas9 & $\begin{array}{l}\text { MATa ura3-52 trp1-289 leu2-3,112 his3 } 11 \text { MAL2-8C } \\
\text { SUC2 } \triangle \text { PEP4 } \triangle \text { PRB1 pCas9 4xUAS-SIS1p-345:uGFP }\end{array}$ & This work \\
\hline sYR93 & $\mathrm{F} 3 \mathrm{~A}$ & TC-77 & $\begin{array}{l}\text { pYR59 } \\
\end{array}$ & - & pCas9 & $\begin{array}{l}\text { MATa ura3-52 trp1-289 leu2-3,112 his3 } 11 \text { MAL2-8C } \\
\text { SUC2 } \triangle \text { PEP4 } \triangle \text { PRB1 pCas9 2xUAS-SSA1p-365:UGFP }\end{array}$ & This work \\
\hline sYR94 & F3A & TC-77 & pYR60 & - & pCas9 & $\begin{array}{l}\text { MATa ura3-52 trp1-289 leu2-3,112 his3 } \triangle 1 \text { MAL2-8C } \\
\text { SUC2 } \triangle \text { PEP4 } \triangle \text { PRB1 pCas9 4xUAS-SSA1p-365:UGFP }\end{array}$ & This work \\
\hline sYR76 & F3A, F3B & TC-77 & pYR45 & - & pCas9 & $\begin{array}{l}\text { MATa ura3-52 trp1-289 leu2-3,112 his3 } 11 \text { MAL2-8C } \\
\text { SUC2 } \triangle \text { PEP4 } \triangle \text { PRB1 pCas9 HSP82p-858:yEGFP3 }\end{array}$ & This work \\
\hline sYR77 & $\mathrm{F} 3 \mathrm{~A}$ & TC-77 & pYR46 & - & pCas9 & $\begin{array}{l}\text { MATa ura3-52 trp1-289 leu2-3,112 his3 } \Delta 1 \text { MAL2-8C } \\
\text { SUC2 } \triangle \text { PEP4 } \triangle \text { PRB1 pCas9 SIS1 p-501:yEGFP3 }\end{array}$ & This work \\
\hline sYR78 & F3A, F3B & TC-77 & $\begin{array}{l}\text { pYR47 } \\
\end{array}$ & - & pCas9 & $\begin{array}{l}\text { MATa ura3-52 trp1-289 leu2-3,112 his3 } 11 \text { MAL2-8C } \\
\text { SUC2 } \triangle \text { PEP4 } \triangle \text { PRB1 pCas9 SSA1p-824:yEGFP3 }\end{array}$ & This work \\
\hline sYR99 & $\mathrm{F} 3 \mathrm{~A}$ & sYR10 & pYR48 & - & pCas9 & $\begin{array}{l}\text { MATa ura3-52 trp1-289 leu2-3,112 his3 } 11 \text { MAL2-8C } \\
\text { SUC2 } \triangle \text { PEP4 } \triangle \text { PRB1 pCas9 TDH3p:his6-mKate2-MBP } \\
\text { 1xUAS-HSP82p-324:uGFP }\end{array}$ & This work \\
\hline sYR101 & F3A & sYR10 & pYR54 & - & pCas9 & $\begin{array}{l}\text { MATa ura3-52 trp1-289 leu2-3,112 his3 } 11 \text { MAL2-8C } \\
\text { SUC2 } \triangle \text { PEP4 } \triangle \text { PRB1 pCas9 TDH3p:his6-mKate2-MBP } \\
\text { 1xUAS-SIS1p-345:uGFP }\end{array}$ & This work \\
\hline sYR102 & F3A & sYR10 & pYR52 & - & pCas9 & $\begin{array}{l}\text { MATa ura3-52 trp1-289 leu2-3,112 his3 } \triangle 1 \text { MAL2-8C } \\
\text { SUC2 } \triangle \text { PEP4 } \triangle \text { PRB1 pCas9 TDH3p:his6-mKate2-MBP } \\
\text { 1xUAS-SSA1p--365:uGFP }\end{array}$ & This work \\
\hline sYR103 & F3A & sYR10 & pYR55 & - & pCas9 & $\begin{array}{l}\text { MATa ura3-52 trp1-289 leu2-3,112 his3 } 1 \text { 1 MAL2-8C } \\
\text { SUC2 } \triangle P E P 4 \triangle P R B 1 \text { pCas9 TDH3p:his6-mKate2-MBP } \\
\text { 2xUAS-HSP82p-324:uGFP }\end{array}$ & This work \\
\hline sYR104 & F3A & sYR10 & pYR56 & - & pCas9 & $\begin{array}{l}\text { MATa ura3-52 trp1-289 leu2-3,112 his3 } 11 \text { MAL2-8C } \\
\text { SUC2 } \triangle \text { PEP4 } \triangle \text { PRB1 pCas9 TDH3p:his6-mKate2-MBP } \\
\text { 4xUAS-HSP82p-324:uGFP }\end{array}$ & This work \\
\hline sYR105 & F3A & sYR10 & pYR57 & - & pCas9 & $\begin{array}{l}\text { MATa ura3-52 trp1-289 leu2-3,112 his3 } \triangle 1 \text { MAL2-8C } \\
\text { SUC2 } \triangle \text { PEP4 } \triangle \text { PRB1 pCas9 TDH3p:his6-mKate2-MBP } \\
\text { 2xUAS-SIS1p-345:uGFP }\end{array}$ & This work \\
\hline sYR106 & F3A & sYR10 & $\begin{array}{l}\text { pYR58 } \\
\end{array}$ & - & pCas9 & $\begin{array}{l}\text { MATa ura3-52 trp1-289 leu2-3,112 his3 } \triangle 1 \text { MAL2-8C } \\
\text { SUC2 } \triangle \text { PEP4 } \triangle \text { PRB1 pCas9 TDH3p:his6-mKate2-MBP } \\
\text { 4xUAS-SIS1p-345:uGFP }\end{array}$ & This work \\
\hline sYR107 & F3A & sYR10 & pYR59 & - & pCas9 & $\begin{array}{l}\text { MATa ura3-52 trp1-289 leu2-3,112 his3 } \triangle 1 \text { MAL2-8C } \\
\text { SUC2 } \triangle \text { PEP4 } \triangle \text { PRB1 pCas9 TDH3p:his6-mKate2-MBP } \\
\text { 2xUAS-SSA1p-365:UGFP }\end{array}$ & This work \\
\hline sYR108 & F3A & sYR10 & pYR60 & - & pCas9 & $\begin{array}{l}\text { MATa ura3-52 trp1-289 leu2-3,112 his3 } 1 \text { MAL2-8C } \\
\text { SUC2 } \triangle \text { PEP4 } \triangle \text { PRB1 pCas9 TDH3p:his6-mKate2-MBP } \\
\text { 4xUAS-SSA1p-365:UGFP }\end{array}$ & This work \\
\hline sYR79 & F3A, F3B & sYR10 & pYR45 & - & pCas9 & $\begin{array}{l}\text { MATa ura3-52 trp1-289 leu2-3,112 his3 } 11 \text { MAL2-8C } \\
\text { SUC2 } \triangle \text { PEP4 } \triangle \text { PRB1 pCas9 TDH3p:his6-mKate2-MBP } \\
\text { HSP82p-858:yEGFP3 }\end{array}$ & This work \\
\hline sYR80 & F3A & sYR10 & pYR46 & - & pCas9 & $\begin{array}{l}\text { MATa ura3-52 trp1-289 leu2-3,112 his3 } 11 \text { MAL2-8C } \\
\text { SUC2 } \triangle \text { PEP4 } \triangle \text { PRB1 pCas9 TDH3p:his6-mKate2-MBP } \\
\text { SIS1p-501:yEGFP3 }\end{array}$ & This work \\
\hline
\end{tabular}




\begin{tabular}{|c|c|c|c|c|c|c|c|}
\hline sYR81 & F3A, F3B & sYR10 & pYR47 & - & pCas9 & $\begin{array}{l}\text { MATa ura3-52 trp1-289 leu2-3,112 his3 } \triangle 1 \text { MAL2-8C } \\
\text { SUC2 } \triangle \text { PEP4 } \triangle \text { PRB1 pCas9 TDH3p:his6-mKate2-MBP } \\
\text { SSA1p-824:yEGFP3 }\end{array}$ & This work \\
\hline sYR113 & F3A & sYR11 & pYR48 & - & pCas9 & $\begin{array}{l}\text { MATa ura3-52 trp1-289 leu2-3,112 his3D1 MAL2-8C } \\
\text { SUC2 } \triangle \text { PEP4 } \triangle \text { PRB1 pCas9 TDH3p:his6-mKate2- } \\
\text { LipPks1 1xUAS-HSP82p-324:uGFP }\end{array}$ & This work \\
\hline sYR115 & F3A & sYR11 & pYR52 & - & pCas9 & $\begin{array}{l}\text { MATa ura3-52 trp1-289 leu2-3,112 his3 } \triangle 1 \text { MAL2-8C } \\
\text { SUC2 } \triangle \text { PEP4 } \triangle \text { PRB1 pCas9 TDH3p:his6-mKate2- } \\
\text { LipPks1 1xUAS-SIS1p-345:uGFP }\end{array}$ & This work \\
\hline SYR116 & $\mathrm{F} 3 \mathrm{~A}$ & sYR11 & pYR54 & - & pCas9 & $\begin{array}{l}\text { MATa ura3-52 trp1-289 leu2-3,112 his3 } 1 \text { MAL2-8C } \\
\text { SUC2 } \triangle \text { PEP4 } \triangle \text { PRB1 pCas9 TDH3p:his6-mKate2- } \\
\text { LipPks1 1xUAS-SSA1p--365:uGFP }\end{array}$ & This work \\
\hline sYR117 & F3A & sYR11 & pYR55 & - & pCas9 & $\begin{array}{l}\text { MATa ura3-52 trp1-289 leu2-3,112 his3 } 1 \text { MAL2-8C } \\
\text { SUC2 } \triangle \text { PEP4 } \triangle \text { PRB1 pCas9 TDH3p:his6-mKate2- } \\
\text { LipPks1 2xUAS-HSP82p-324:uGFP }\end{array}$ & This work \\
\hline sYR118 & F3A & sYR11 & pYR56 & - & pCas9 & $\begin{array}{l}\text { MATa ura3-52 trp1-289 leu2-3,112 his3D1 MAL2-8C } \\
\text { SUC2 } \triangle \text { PEP4 } \triangle \text { PRB1 pCas9 TDH3p:his6-mKate2- } \\
\text { LipPks1 4xUAS-HSP82p-324:uGFP }\end{array}$ & This work \\
\hline sYR119 & F3A & sYR11 & pYR57 & - & pCas9 & $\begin{array}{l}\text { MATa ura3-52 trp1-289 leu2-3,112 his3 } \triangle 1 \text { MAL2-8C } \\
\text { SUC2 } \triangle \text { PEP4 } \triangle \text { PRB1 pCas9 TDH3p:his6-mKate2- } \\
\text { LipPks1 2xUAS-SIS1p-345:uGFP }\end{array}$ & This work \\
\hline sYR120 & F3A & sYR11 & pYR58 & - & pCas9 & $\begin{array}{l}\text { MATa ura3-52 trp1-289 leu2-3,112 his3 } \triangle 1 \text { MAL2-8C } \\
\text { SUC2 } \triangle \text { PEP4 } \triangle \text { PRB1 pCas9 TDH3p:his6-mKate2- } \\
\text { LipPks1 4xUAS-SIS1p-345:uGFP }\end{array}$ & This work \\
\hline SYR121 & F3A & sYR11 & pYR59 & - & pCas9 & $\begin{array}{l}\text { MATa ura3-52 trp1-289 leu2-3,112 his3 } 11 \text { MAL2-8C } \\
\text { SUC2 } \triangle \text { PEP4 } \triangle \text { PRB1 pCas9 TDH3p:his6-mKate2- } \\
\text { LipPks1 2xUAS-SSA1p-365:uGFP }\end{array}$ & This work \\
\hline sYR122 & F3A & sYR11 & pYR60 & - & pCas9 & $\begin{array}{l}\text { MATa ura3-52 trp1-289 leu2-3,112 his3D1 MAL2-8C } \\
\text { SUC2 } \triangle \text { PEP4 } \triangle \text { PRB1 pCas9 TDH3p:his6-mKate2- } \\
\text { LipPks1 4xUAS-SSA1p--365:uGFP }\end{array}$ & This work \\
\hline sYR82 & F3A, F3B & sYR11 & pYR45 & - & pCas9 & $\begin{array}{l}\text { MATa ura3-52 trp1-289 leu2-3,112 his3 } 11 \text { MAL2-8C } \\
\text { SUC2 } \triangle \text { PEP4 } \triangle \text { PRB1 pCas9 TDH3p:his6-mKate2- } \\
\text { LipPs1 HSP82p-858:yEGFP3 }\end{array}$ & This work \\
\hline sYR83 & $\mathrm{F} 3 \mathrm{~A}$ & sYR11 & pYR46 & - & pCas9 & $\begin{array}{l}\text { MATa ura3-52 trp1-289 leu2-3,112 his3 } 1 \text { MAL2-8C } \\
\text { SUC2 } \triangle \text { PEP4 } \triangle \text { PRB1 pCas9 TDH3p:his6-mKate2- } \\
\text { LipPks1 SIS1p-501:yEGFP3 }\end{array}$ & This work \\
\hline sYR84 & F3A, F3B & sYR11 & pYR47 & - & pCas9 & $\begin{array}{l}\text { MATa ura3-52 trp1-289 leu2-3,112 his3 } \Delta 1 \text { MAL2-8C } \\
\text { SUC2 } \triangle \text { PEP4 } \triangle \text { PRB1 pCas9 TDH3p:his6-mKate2- } \\
\text { LipPks1 SSA1p-824:yEGFP3 }\end{array}$ & This work \\
\hline sDRS432 & F3B & TC-77 & pDRS86 & - & pCas9 & $\begin{array}{l}\text { MATa ura3-52 trp1-289 leu2-3,112 his3 } \triangle 1 \text { MAL2-8C } \\
\text { SUC2 } \triangle \text { PEP4 } \triangle \text { PRB1 pCas9 2xUAS-HSP82p- } \\
\text { 324:yEGFP3 }\end{array}$ & This work \\
\hline SYR127 & F3B & TC-77 & pYR65 & - & pCas9 & $\begin{array}{l}\text { MATa ura3-52 trp1-289 leu2-3,112 his3 } \triangle 1 \text { MAL2-8C } \\
\text { SUC2 } \triangle \text { PEP4 } \triangle \text { PRB1 pCas9 4xUAS-HSP82p- } \\
\text { 324:yEGFP3 }\end{array}$ & This work \\
\hline SYR128 & F3B & TC-77 & pYR67 & - & pCas9 & $\begin{array}{l}\text { MATa ura3-52 trp1-289 leu2-3,112 his3 } 11 \text { MAL2-8C } \\
\text { SUC2 } \triangle \text { PEP4 } \triangle \text { PRB1 pCas9 2xUAS-SSA1p-365:yEGFP3 }\end{array}$ & This work \\
\hline SYR129 & $\begin{array}{l}\text { F3B, F4A, } \\
\text { f4B, F4C, } \\
\text { F4D, F4G }\end{array}$ & TC-77 & pYR69 & - & pCas9 & $\begin{array}{l}\text { MATa ura3-52 trp1-289 leu2-3,112 his3 } 1 \text { MAL2-8C } \\
\text { SUC2 } \triangle \text { PEP4 } \triangle \text { PRB1 pCas9 4xUAS-SSA1p-365:VEGFP3 }\end{array}$ & This work \\
\hline sDRS433 & F3B & sYR10 & pDRS86 & - & pCas9 & $\begin{array}{l}\text { MATa ura3-52 trp1-289 leu2-3,112 his3 } 1 \text { MAL2-8C } \\
\text { SUC2 } \triangle \text { PEP4 } \triangle \text { PRB1 pCas9 TDH3p:his6-mKate2-MBP } \\
\text { 2xUAS-HSP82p-324:yEGFP3 }\end{array}$ & This work \\
\hline sYR130 & F3B & sYR10 & pYR65 & - & pCas9 & $\begin{array}{l}\text { MATa ura3-52 trp1-289 leu2-3,112 his3 } 1 \text { MAL2-8C } \\
\text { SUC2 } \triangle \text { PEP4 } \triangle \text { PRB1 pCas9 TDH3p:his6-mKate2-MBP } \\
\text { 4xUAS-HSP82p-324:yEGFP3 }\end{array}$ & This work \\
\hline sYR131 & F3B & sYR10 & pYR67 & - & pCas9 & 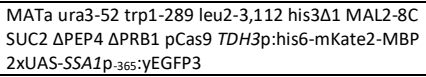 & This work \\
\hline sYR132 & $\begin{array}{l}\text { F3B, F4A, } \\
\text { F4B, F4C, } \\
\text { F4D, SF12 }\end{array}$ & sYR10 & pYR69 & - & pCas9 & $\begin{array}{l}\text { MATa ura3-52 trp1-289 leu2-3,112 his3 } \triangle 1 \text { MAL2-8C } \\
\text { SUC2 } \triangle \text { PEP4 } \triangle \text { PRB1 pCas9 TDH3p:his6-mKate2-MBP } \\
\text { 4xUAS-SSA1p-365:yEGFP3 }\end{array}$ & This work \\
\hline sDRS434 & F3B & sYR11 & pDRS86 & - & pCas9 & $\begin{array}{l}\text { MATa ura3-52 trp1-289 leu2-3,112 his3D1 MAL2-8C } \\
\text { SUC2 } \triangle \text { PEP4 } \triangle \text { PRB1 pCas9 TDH3p:his6-mKate2- } \\
\text { LipPks1 2xUAS-HSP82p-324:yEGFP3 }\end{array}$ & This work \\
\hline sYR133 & F3B & sYR11 & pYR65 & - & pCas9 & $\begin{array}{l}\text { MATa ura3-52 trp1-289 leu2-3,112 his3 } 1 \text { MAL2-8C } \\
\text { SUC2 } \triangle \text { PEP4 } \triangle \text { PRB1 pCas9 TDH3p:his6-mKate2- } \\
\text { LipPks1 4xUAS-HSP82p-324:yEGFP3 }\end{array}$ & This work \\
\hline sYR134 & F3B & sYR11 & pYR67 & - & pCas9 & $\begin{array}{l}\text { MATa ura3-52 trp1-289 leu2-3,112 his3D1 MAL2-8C } \\
\text { SUC2 } \triangle \text { PEP4 } \triangle \text { PRB1 pCas9 TDH3p:his6-mKate2- } \\
\text { LipPks1 2xUAS-SSA1p--365:yEGFP3 }\end{array}$ & This work \\
\hline SYR135 & $\begin{array}{l}\text { F3B, F4A, } \\
\text { F4B, F4C, } \\
\text { F4D, F4E, } \\
\text { F4F, SF12 }\end{array}$ & sYR11 & pYR69 & - & pCas9 & $\begin{array}{l}\text { MATa ura3-52 trp1-289 leu2-3,112 his3 } \triangle 1 \text { MAL2-8C } \\
\text { SUC2 } \triangle \text { PEP4 } \triangle \text { PRB1 pCas9 TDH3p:his6-mKate2- } \\
\text { LipPks1 4xUAS-SSA1p-365:yEGFP3 }\end{array}$ & This work \\
\hline sDRS444 & F4A, F4B & sYR129 & pYR69 & aDRS349 & pCas9 & $\begin{array}{l}\text { MATa ura3-52 trp1-289 leu2-3,112 his3 } \triangle 1 \text { MAL2-8C } \\
\text { SUC2 } \triangle \text { PEP4 } \triangle \text { PRB1 pCas9 TEF1p:his6-mKate2-MBP } \\
\text { 4xUAS-SSA1p-365:yEGFP3 }\end{array}$ & This work \\
\hline sDRS435 & F4A, F4B & sYR129 & pYR69 & aDRS346 & pCas9 & $\begin{array}{l}\text { MATa ura3-52 trp1-289 leu2-3,112 his3 } \triangle 1 \text { MAL2-8C } \\
\text { SUC2 } \triangle \text { PEP4 } \triangle \text { PRB1 pCas9 PGK1p:his6-mKate2-MBP } \\
\text { 4xUAS-SSA1p-365:yEGFP3 }\end{array}$ & This work \\
\hline sDRS436 & F4A, F4B & sYR129 & $\begin{array}{l}\text { pYR69, } \\
\text { pDRS61 }\end{array}$ & - & pCas9 & $\begin{array}{l}\text { MATa ura3-52 trp1-289 leu2-3,112 his3 } 1 \text { MAL2-8C } \\
\text { SUC2 } \triangle \text { PEP4 } \triangle \text { PRB1 pCas9 RPL18Bp:his6-mKate2- } \\
\text { MBP 4xUAS-SSA1p-365:yEGFP3 }\end{array}$ & This work \\
\hline sDRS437 & F4A, F4B & sYR129 & pYR69 & aDRS347 & pCas9 & $\begin{array}{l}\text { MATa ura3-52 trp1-289 leu2-3,112 his3 } 11 \text { MAL2-8C } \\
\text { SUC2 } \triangle \text { PEP4 } \triangle \text { PRB1 pCas9 RNR2p:his6-mKate2-MBP } \\
\text { 4xUAS-SSA1p-365:yEGFP3 }\end{array}$ & This work \\
\hline sDRS438 & F4A, F4B & SYR129 & $\begin{array}{l}\text { pYR69, } \\
\text { pDRS63 }\end{array}$ & - & pCas9 & $\begin{array}{l}\text { MATa ura3-52 trp1-289 leu2-3,112 his3D1 MAL2-8C } \\
\text { SUC2 } \triangle \text { PEP4 } \triangle \text { PRB1 pCas9 REV1p:his6-mKate2-MBP } \\
\text { 4xUAS-SSA1p--365:yEGFP3 }\end{array}$ & This work \\
\hline sDRS443 & F4A, F4B & sYR129 & pYR69 & aDRS348 & pCas9 & $\begin{array}{l}\text { MATa ura3-52 trp1-289 leu2-3,112 his3D1 MAL2-8C } \\
\text { SUC2 } \triangle \text { PEP4 } \triangle \text { PRB1 pCas9 TEF1p:his6-mKate2- } \\
\text { LipPs1 2xUAS-HSP82p-324:yEGFP3 }\end{array}$ & This work \\
\hline
\end{tabular}




\begin{tabular}{|c|c|c|c|c|c|c|c|}
\hline sDRS439 & F4A, F4B & sYR129 & pYR69 & aDRS342 & pCas9 & $\begin{array}{l}\text { MATa ura3-52 trp1-289 leu2-3,112 his3 } 1 \text { MAL2-8C } \\
\text { SUC2 } \triangle \text { PEP4 } \triangle \text { PRB1 pCas9 PGK1p:his6-mKate2- } \\
\text { LipPks1 2xUAS-HSP82p-324:yEGFP3 }\end{array}$ & This work \\
\hline sDRS440 & F4A, F4B & SYR129 & pYR69 & aDRS343 & pCas9 & $\begin{array}{l}\text { MATa ura3-52 trp1-289 leu2-3,112 his3 } 11 \text { MAL2-8C } \\
\text { SUC2 } \triangle \text { PEP4 } \triangle \text { PRB1 pCas9 RPL18Bp:his6-mKate2- } \\
\text { LipPks1 2xUAS-HSP82p-324:yEGFP3 }\end{array}$ & This work \\
\hline sDRS441 & F4A, F4B & sYR129 & pYR69 & aDRS344 & pCas9 & $\begin{array}{l}\text { MATa ura3-52 trp1-289 leu2-3,112 his3 } 11 \text { MAL2-8C } \\
\text { SUC2 } \triangle \text { PEP4 } \triangle \text { PRB1 pCas9 RNR2p:his6-mKate2- } \\
\text { LipPks1 2xUAS-HSP82p-324:yEGFP3 }\end{array}$ & This work \\
\hline sDRS442 & F4A, F4B & SYR129 & pYR69 & aDRS345 & pCas9 & $\begin{array}{l}\text { MATa ura3-52 trp1-289 leu2-3,112 his3D1 MAL2-8C } \\
\text { SUC2 } \triangle P E P 4 \triangle P R B 1 \text { pCas9 REV1p:his6-mKate2- } \\
\text { LipPks1 2xUAS-HSP82p-324:yEGFP3 }\end{array}$ & This work \\
\hline sDRS468 & F4G & sYR129 & pYR69 & aDRS354 & pCas9 & $\begin{array}{l}\text { MATa ura3-52 trp1-289 leu2-3,112 his3D1 MAL2-8C } \\
\text { SUC2 } \triangle \text { PEP4 } \triangle \text { PRB1 pCas9 4xUAS-SSA1p. } \\
\text { 365:yEGFP3XII-5 TDH3p:his6-mKate2-SUP35 }\end{array}$ & This work \\
\hline sDRS469 & F4G & SYR129 & pYR69 & aDRS355 & pCas9 & $\begin{array}{l}\text { MATa ura3-52 trp1-289 leu2-3,112 his3D1 MAL2-8C } \\
\text { SUC2 } \triangle \text { PEP4 } \triangle \text { PRB1 pCas9 4xUAS-SSA1p. } \\
\text { 365:yEGFP3XII-5 TDH3p:his6-mKate2-RNQ1 }\end{array}$ & This work \\
\hline sDRS471 & F4G & SYR129 & pYR69 & aDRS357 & pCas9 & $\begin{array}{l}\text { MATa ura3-52 trp1-289 leu2-3,112 his3 } 11 \text { MAL2-8C } \\
\text { SUC2 } \triangle \text { PEP4 } \triangle \text { PRB1 pCas9 4xUAS-SSA1p. } \\
\text { 365:yEGFP3XII-5 TDH3p:his6-mKate2-CYC8 }\end{array}$ & This work \\
\hline sYR151 & $\mathrm{F} 4 \mathrm{I}$ & CEN.PK2-1C & pYR70 & aDRS247 & pCas9 & $\begin{array}{l}\text { MATa ura3-52 trp1-289 leu2-3,112 his3D1 MAL2-8C } \\
\text { SUC2 pCas9 4xUAS-SSA1p-365:mKate2 } \\
\text { TDH3p:YFPm4-HIS5 }\end{array}$ & This work \\
\hline sDRS423 & F4I & TC-77 & pYR70 & aDRS318 & pCas9 & $\begin{array}{l}\text { MATa ura3-52 trp1-289 leu2-3,112 his3 } 11 \text { MAL2-8C } \\
\text { SUC2 } \triangle \text { PEP4 } \triangle \text { PRB1 pCas9 4xUAS-SSA1p-365: mKate2 } \\
\text { TDH3p:his6-yEGFP3 }\end{array}$ & This work \\
\hline sDRS472 & SF15 & sDRS23 & pYR69 & - & pCas9 & $\begin{array}{l}\text { MATa ura3-52 trp1-289 leu2-3,112 his3 } 11 \text { MAL2-8C } \\
\text { SUC2 pCas9 4xUAS-SSA1p--365:yEGFP3 }\end{array}$ & This work \\
\hline sDRS473 & SF15 & sDRS23 & $\begin{array}{l}\text { pYR69, } \\
\text { pYR10 }\end{array}$ & - & pCas9 & $\begin{array}{l}\text { MATa ura3-52 trp1-289 leu2-3,112 his3D1 MAL2-8C } \\
\text { SUC2 pCas9 TDH3p:his6-mKate2-MBP 4xUAS- } \\
\text { SSA1p-365:yEGFP3 }\end{array}$ & This work \\
\hline sDRS474 & SF15 & sDRS23 & $\begin{array}{l}\text { pYR69, } \\
\text { pYR11 }\end{array}$ & - & pCas9 & $\begin{array}{l}\text { MATa ura3-52 trp1-289 leu2-3,112 his3 } 1 \text { MAL2-8C } \\
\text { SUC2 pCas9 TDH3p:his6-mKate2-LipPks1 4xUAS- } \\
\text { SSA1p-365:yEGFP3 }\end{array}$ & This work \\
\hline sDRS479 & SF7 & sYR10 & - & aDRS371 & pCas9 & $\begin{array}{l}\text { MATa ura3-52 trp1-289 leu2-3,112 his3D1 MAL2-8C } \\
\text { SUC2 } \triangle \text { PEP4 } \triangle \text { PRB1 pCas9 TDH3p:his6-mKate2-MBP } \\
\text { HSP104:yEGFP3-HIS5 }\end{array}$ & This work \\
\hline sDRS480 & SF7 & sYR11 & - & aDRS371 & pCas9 & $\begin{array}{l}\text { MATa ura3-52 trp1-289 leu2-3,112 his3 } 11 \text { MAL2-8C } \\
\text { SUC2 } \triangle \text { PEP4 } \triangle \text { PRB1 pCas9 TDH3p:his6-mKate2- } \\
\text { LipPks1 HSP104:yEGFP3-HIS5 }\end{array}$ & This work \\
\hline
\end{tabular}

\section{Supplementary Table 4. Gene sequences used in this study}

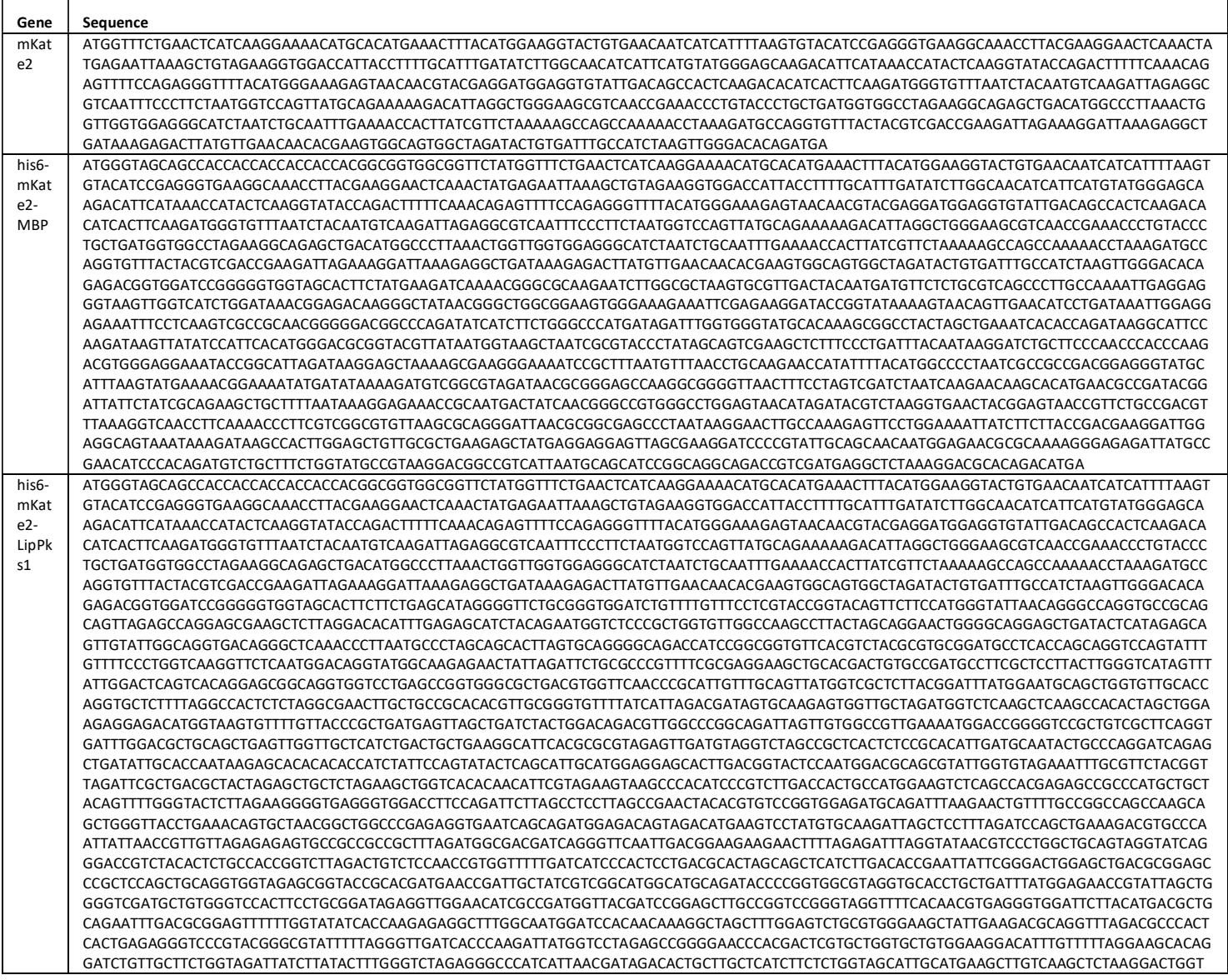




\begin{tabular}{|c|c|}
\hline & 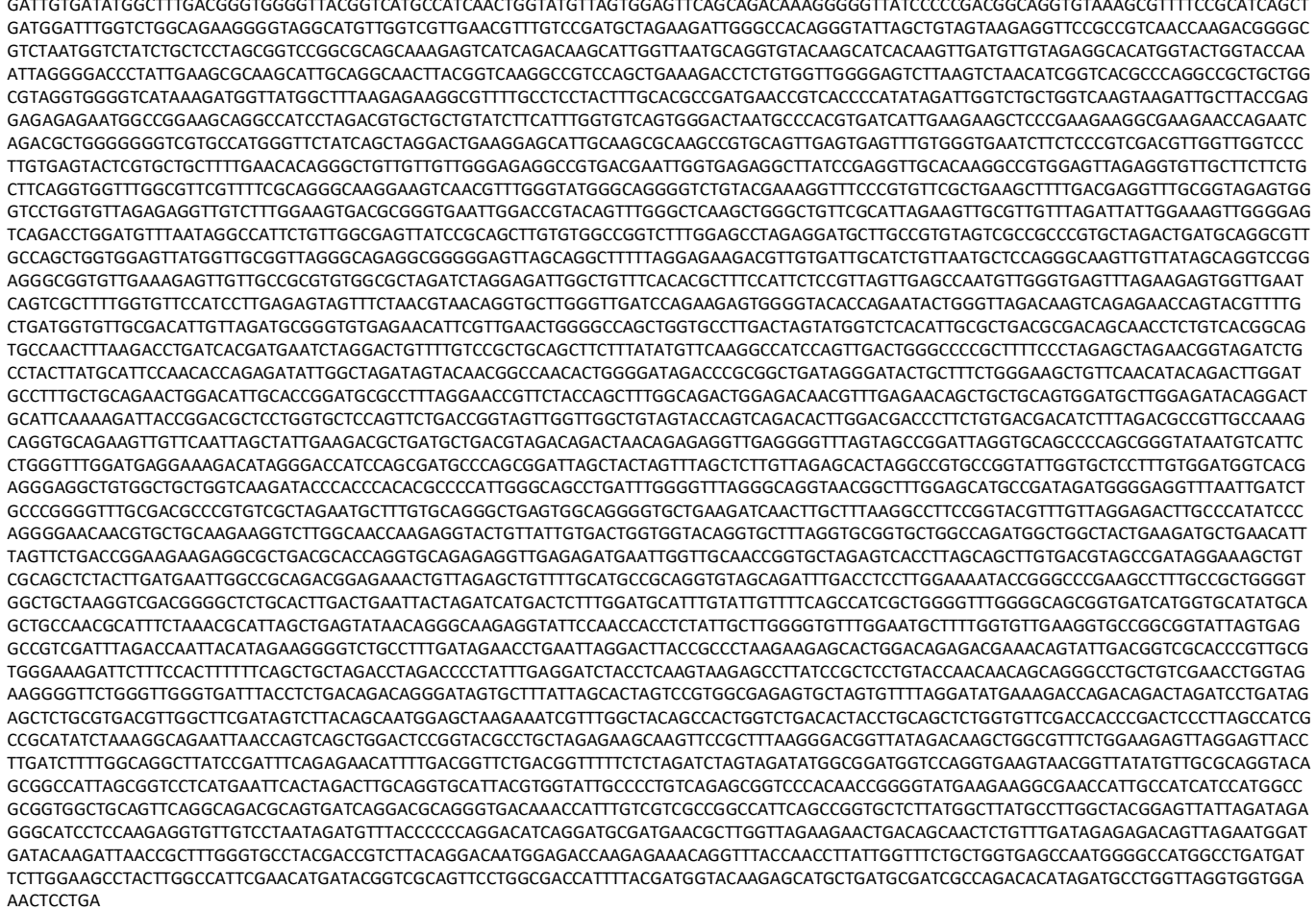 \\
\hline & 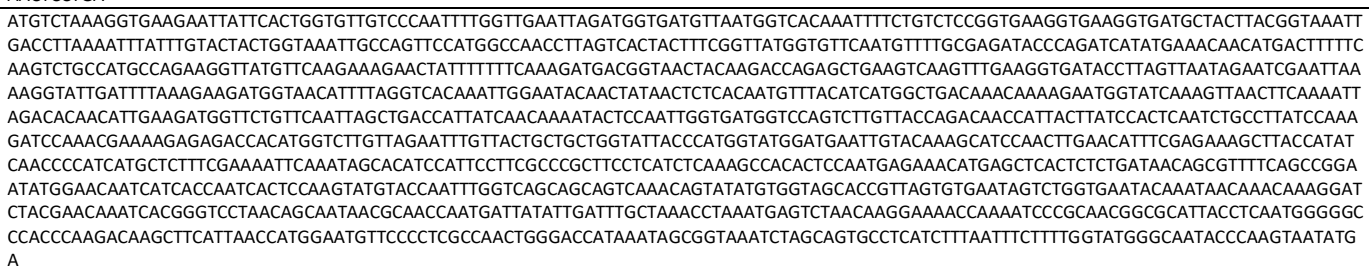 \\
\hline $\begin{array}{l}\text { yEGF } \\
\text { P3 }\end{array}$ & 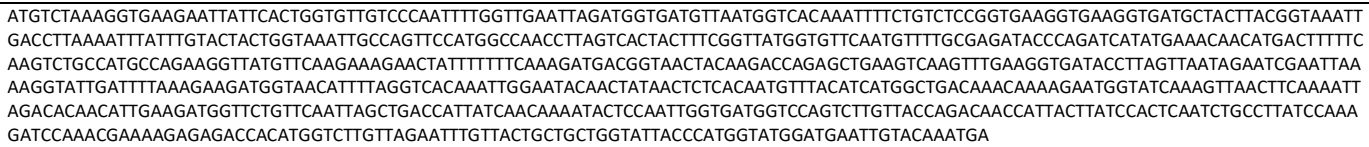 \\
\hline & 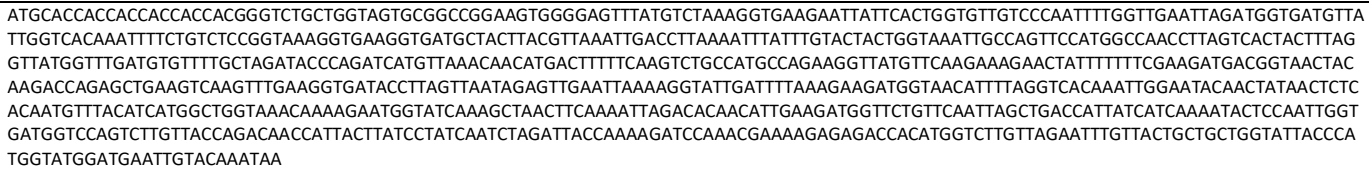 \\
\hline $\begin{array}{l}\text { his6- } \\
\text { yEGF } \\
\text { P3 }\end{array}$ & 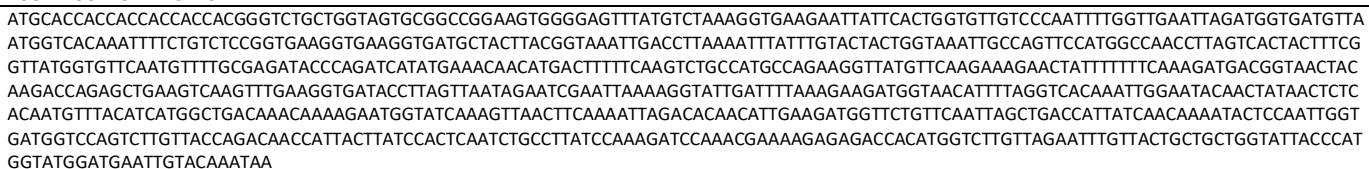 \\
\hline & 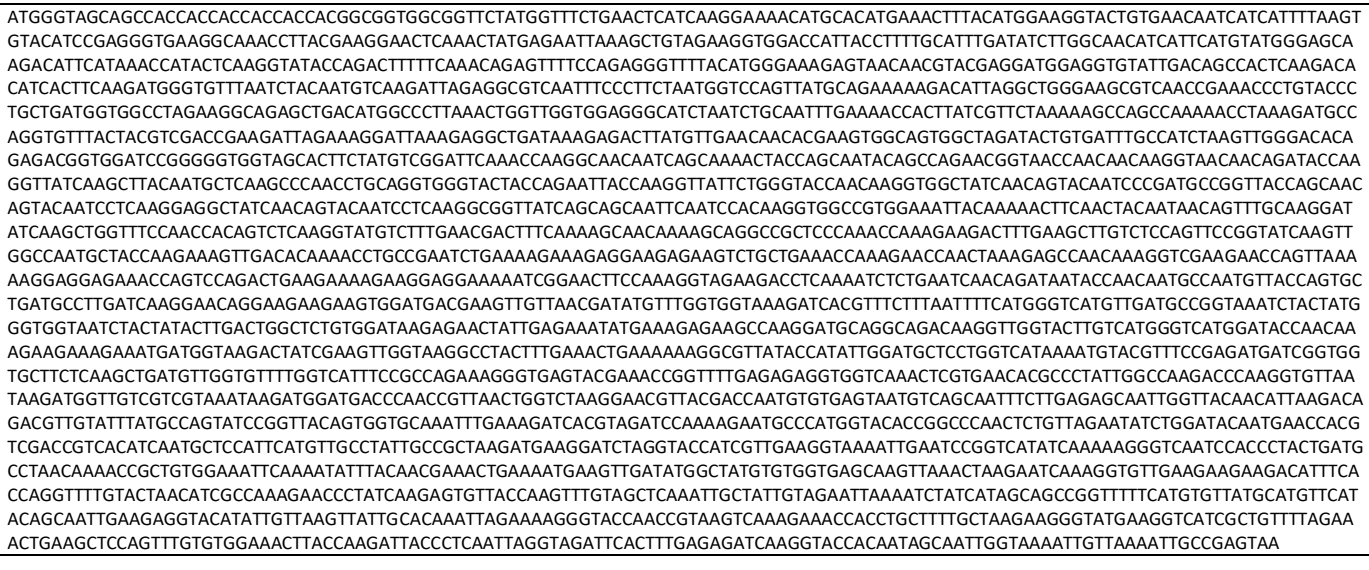 \\
\hline
\end{tabular}


his6- | ATGGGTAGCAGCCACCACCACCACCACCACGGCGGTGGCGGTTCTATGGTTTCTGAACTCATCAAGGAAAACATGCACATGAAACTTTACATGGAAGGTACTGTGAACAATCATCATTTTAAGT mKat GTACATCCGAGGGTGAAGGCAACCTTACGAAGGACTCAAACTATGAGAATTAAAGCTGTAGAAGGTGGACCATTACCTTTTGCATTGATATCTTGGCAACATCATTCATGTATGGGAC

R TGCTGATGGTGGCCTAGAAGGCAGAGCTGACATGGCCCTTAAACTGGTTGGTGGAGGGCATCTAATCTGCAATTTGAAAACCACTTATCGTTCTAAAAAGCCAGCCAAAAACCTAAAGATGCC AGGTGTTIACTACGTCGACCGAAGATTAGAAAGGATTAAAGAGGCTGATAAAGAGACTTATGTTGAACAACACGAAGTGGCAGTGGCTAGATACTGTGATTTGCCATCTAAGTTGGGACACA GAGACGGTGGATCCGGGGGTGGTAGCACTTCTATGGATACGGATAAGTTAATCTCAGAGGCTGAGTCTCATTTITCTCAAGGAAACCATGCAGAAGCTGTTGCGAAGTTGACATCCGCAGCTC AGTCGAACCCCAATGACGAGCAAATGTCAACTATTGAATCATTAATTCAAAAAATCGCAGGATACGTCATGGACAACCGTAGTGGTGGTAGTGACGCCTCGCAAGATCGTGCTGCTGGTGGTG GTTCATCTTTTATGAACACTTTAATGGCAGACTCTAAGGGTTCTTCCCAAACGCAACTAGGAAAACTAGCTTTGTTAGCCACAGTGATGACACACTCATCAAATAAAGGTTCTTCTAACAGAGGG TITGACGTAGGGACTGTCATGTCAATGCTAAGTGGTTCTGGCGGCGGGAGCCAAAGTATGGGTGCTTCCGGCCTGGCTGCCTTGGCTTCTCAATTCTTTAAGTCAGGTAACAATTCCCAAGGTC AGGGACAAGGTCAAGGTCAAGGTCAAGGTCAAGGACAAGGTCAAGGTCAAGGTTCTTTTACTGCTTTGGCGTCTTTGGCTTCATCTTTCATGAATTCCAACAACAATAATCAGCAAGGTCAAAA TCAAAGCTCCGGTGGTTCTTCCTTIGGAGCACTGGCTTCTATGGCAAGCTCTTTTATGCATTCCAATAATAATCAGAACTCCAACAATAGTCAACAGGGCTATAACCAATCCTATCAAAACGGTA ACCAAAATAGTCAAGGTIACAATAATCAACAGTACCAAGGTGGCAACGGTGGTTACCAACAACAACAGGGACAATCTGGTGGTGCTTTTTCCTCATTGGCCTCCATGGCTCAATCTTACTTAGG TGGTGGACAAACTCAATCCAACCAACAGCAATACAATCAACAAGGCCAAAACAACCAGCAGCAATACCAGCAACAAGGCCAAAACTATCAGCATCAACAACAGGGTCAGCAGCAGCAACAAG GCCACTCCAGTTCATTCTCAGCTTTGGCTTCCATGGCAAGTTCCTACCTGGGCAATAACTCCAATTCAAATTCGAGTTATGGGGGCCAGCAACAGGCTAATGAGTATGGTAGACCGCAACAGAA TGGTCAACAGCAATCCAATGAGTACGGAAGACCGCAATACGGCGGAAACCAGAACTCCAATGGACAGCACGAATCCTTCAATTITTCTGGCAACTTITCTCAACAGAACAATAACGGCAACCA GAACCGCTACTGA

his6- $\quad$ ATGGGTAGCAGCCACCACCACCACCACCACGGCGGTGGCGGTTCTATGGTTTCTGAACTCATCAAGGAAAACATGCACATGAAACTTTACATGGAAGGTACTGTGAACAATCATCATTTTAAGT mkat GTACATCCGAGGGTGAAGGCAAACCTTACGAAGGAACTCAAACTATGAGAATTAAAGCTGTAGAAGGTGGACCATTACCTTTTGCATTTGATATCTTGGCAACATCATTCATGTATGGGAGCA e2- $\quad$ AGACATTCATAAACCATACTCAAGGTATACCAGACTTTTTCAAACAGAGTTTTCAGAGGGTTTTACATGGGAAAGAGTAACAACGTACGAGGATGGAGGTGTATTGACAGCCACTCAAGACA

CYC8 CATCACTTCAAGATGGGTGTTTAATCTACAATGTCAAGATTAGAGGCGTCAATTTCCCTTCTAATGGTCCAGTTATGCAGAAAAAGACATTAGGCTGGGAAGCGTCAACCGAAACCCTGTACCC TGCTGATGGTGGCCTAGAAGGCAGAGCTGACATGGCCCTTAAACTGGTTGGTGGAGGGCATCTAATCTTGCAATTGAAAACCACTTATCGTTCTAAAAAGCCAGCCAAAACCTAAAGATGCC AGGTTGTTACTACGTCGACCGAAGATTAGAAAGGATTAAAGAGGCTGATAAAGAGACTTATGTGAACAACACGAAGTGGCAGTGGCTAGATACTGTGATTGCCATCTAAGTTGGGACACA GAGACGGTGGATCCGGGGGTGGTAGCACTTCTATGAATCCGGGCGGTGAACAAACAATAATGGAACAACCCGCTCAACAGCAACAACAACAGCAACAACAACAGCAGCAACAGCAACAGCA GGCAGCAGTTCCTCAGCAGCCACTCGACCCATTAACACAATCAACTGCGGAAACTTGGCTCTCCATTGCTTCTTTGGCAGAAACCCTTGGTGATGGCGACAGGGCCGCAATGGCATATGACGCC ACTTTACAGTTCAATCCCTCATCTGCAAAGGCTTTAACATCTTTGGCTCACTTGTACCGTTCCAGAGACATGTTCCAAAGAGCTGCAGAATTATATGAAAGAGCACTTTTGGTAAATCCCGAACTA TCAGATGTGTGGGCTACTTTAGGTCATTGTTATCTGATGCTGGATGATCTGCAAAGAGCTTACAATGCCTATCAACAGGCTCTCTACCACCTCAGTAATCCCAACGTACCGAAATTATGGCATGG AATCGGCATTCTTTATGACAGATATGGTTCGCTCGACTATGCCGAAGAAGCTTTTGCCAAAGTTTTGGAATTGGACCCTCATTTTGAAAAGGCAAACGAAATTTACTTCAGACTAGGTATTATTT ATAAACATCAGGGTAAATGGTCTCAAGCTTTGGAATGCTTCAGATACATTCTCCCTCAACCTCCTGCTCCCTTGCAGGAGTGGGACATATGGTTTCAGTTGGGTAGTGTTTTGGAGAGTATGGG AGAGTGGCAAGGTGCGAAGGAAGCCTACGAGCATGTCTTGGCTCAAAATCAACATCATGCCAAAGTATTACAACAATTAGGTTGTCTTTACGGTATGAGTAACGTACAATTTTATGACCCTCAA AAGGCATTGGATTATCTTCTAAAGTCGTTAGAAGCAGATCCCTCCGATGCCACTACATGGTACCATCTCGGTAGAGTGCATATGATTAGAACAGATTATACTGCCGCATATGATGCTTTCCAACA AGCTGTTAATAGAGATTCAAGAAACCCTATCTTTTGGTGCTCAATCGGTGTTTATATTACCAAATTTTCAATACAGAGACGCCTTAGACGCGTACACAAGAGCCATAAGATTAAATCCTTATAT TAGTGAAGTTTGGTACGATCTAGGTACTCTTACGAAACTTGTAACAACCAATTATCTGACGCCCTTGATGCGTATAAGCAAGCTGCAAGACTGGACGTAAATAATGTTCACATAAGAGAAAGA TTAGAAGCTTTAACAAAGCAGTTAGAAAACCCAGGCAATATAAACAAATCGAACGGTGCGCCAACGAATGCCTCTCCTGCCCCACCTCCTGTGATTTIACAACCTACCTTACAACCTAATGATCA AGGAAATCCTTTGAACACTAGAATTTCAGCCCAATCTGCCAATGCTACTGCTTCAATGGTACAACAACAGCATCCTGCTCAACAAACGCCTATTAACTCTTCTGCAACAATGTACAGTAATGGAG CTTCCCCTCAATTACAAGCTCAAGCTCAAGCTCAAGCTCAAGCACAAGCTCAAGCACAAGCACAAGCTCAAGCACAAGCACAAGCACAAGCGCAAGCACAAGCACAAGCACAGGCGCAAGCA CAGGCACAAGCACAAGCACAAGCACATGCACAAGCGCAAGCACAAGCACAAGCACAGGCACAAGCACAAGCACAGGCGCAGGCACAACAACAACAACAACAACAGCAACAACAACAACAAC AACAACAACAACAACAACAACAACAACAACAACAACAACAACAACAGCAGCAGCAATTACAGCCCCTACCAAGACAACAGCTGCAGCAAAAGGGAGTTTCTGTGCAAATGTTAAATCCTCAAC AAGGGCAACCATATATCACACAGCCAACAGTCATACAAGCTCACCAACTGCAACCATTTTCTACACAAGCTATGGAACATCCGCAAAGCTCTCAACTGCCACCTCAACAGCAACAACTACAATCT GTTCAACATCCACAACAACTTCAAGGCCAGCCTCAAGCCCAAGCTCCCCAACCTTTAATCCAGCATAACGTGGAACAGAACGTTTTACCTCAAAAGAGATACATGGAAGGTGCAATCCACACT TAGTAGATGCCGCCGTATCCAGTAGCACCCACACAGAGAATAACACAAAGTCTCCTCGTCAACCAACCCATGCCATTCCAACGCAAGCTCCCGCAACAGGAATAACGAACGCTGAACCACAGG TAAAGAAGCAAAAGTTGAACTCTCCAAATTCAAACATCAACAAATTAGTAAATACTGCTACTTCCATTGAAGAAAATGCAAAATCTGAGGTGAGCAACCAATCGCCAGCAGTAGTGGAGTCTAA TACCAATAATACTTCACAAGAAGAAAAACCTGTAAAAGCAAACTCAATACCTTCAGTAATTGGCGCACAGGAACCTCCACAGGAAGCTAGTCCTGCTGAAGAAGCTACCAAAGCAGCTTCTGTT TCTCCTTCTACAAAACCGCTTAATACGGAACCAGAGTCATCTAGTGTCCAACCAACTGTATCATCAGAAAGTTCAACAACAAAAGCAAATGACCAAAGCACTGCTGAGACCATAGAACTTTCTAC TGCTACTGTTCCTGCAGAAGCAAGCCCTGTAGAAGACGAAGTAAGACAGCATTCTAAAGAGGAAACGGCACAACTGAAGCATCTGCACCTTCTACTGAAGAGGCGGAGCCAGCAGCTTCCA GAGATGCTGAAAAACAACAAGATGAAACCGCTGCTACAACGATAACTGTAATCAAACCTACTTTGGAAACAATGGAAACAGTGAAAGAGGAGGCCAAAATGCGTGAGGAAGAGCAAACATC TCAAGAAAAATCCCCACAGGAGAACACACTTCCAAGAGAAAATGTAGTAAGGCAAGTGGAAGAAGATGAAAACTACGACGACTAA

\section{Supplementary Table 5. Promoters sequences used in this study}

\begin{tabular}{|c|c|}
\hline Promoter & Sequence \\
\hline AHA1p $\mathrm{p}_{-382}$ & 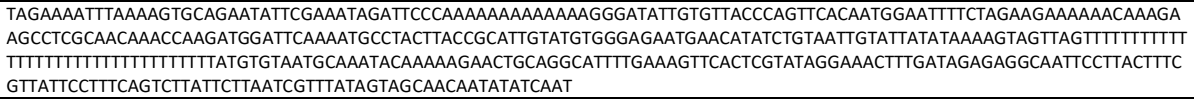 \\
\hline CPR6p-254 & $\begin{array}{l}\text { AAATCTAAATGTCGTGATGTATAACTTGTATATAATAGACAGCTGCAGTGATCGAAACACATTGTTTCCCTTTATAGAACATAACTGTTACGCTTTTGAACGGCATTTCT } \\
\text { ATGAGCTTCTAGAATATTTITCCGCCCTAGCTGGAGAAAGTTCAGACAGAAAATTATTTAAATAAGTCGAATATCAGAGGTGCTGATGCGCTCACATCACATAGAAAA } \\
\text { CTGGTAAGACAATATTCAGGCGATCAAGGAGTAAAA }\end{array}$ \\
\hline$H S C 82 p_{-384}$ & $\begin{array}{l}\text { CCCATTACGCATTTGATTATAATTTGCTTCTTAGGCAAAATTAATATTTACGTTCTTTTATATTCTTTCTTTTTGTATTCATAGAACAGCAGCCATTACCAATAGAAAGTTA } \\
\text { AAATAGCCGCCGATGCATTTATTACCCGCCTTCTGTTTCTGGGCACTTTCTTTCTAGAAGGTGAAAGAACAATTTTCTCGTTTTCTCGAACTTCCACCAAGCGTTG } \\
\text { GGTAATGAGGGAGGAGATTTGTATAAAAAGAGTGGCATGTGAACTGCCTACCGTAAGTGACATGAACACATGCATTATATTTTTGTGATATATTCTTCTCTTGTTTT } \\
\text { CTTITTCTTGAAACGCTACAGAACCAATAGAAAAATAGAATCATTCTGAAAT }\end{array}$ \\
\hline HSP42 $\mathrm{p}_{-1000}$ & 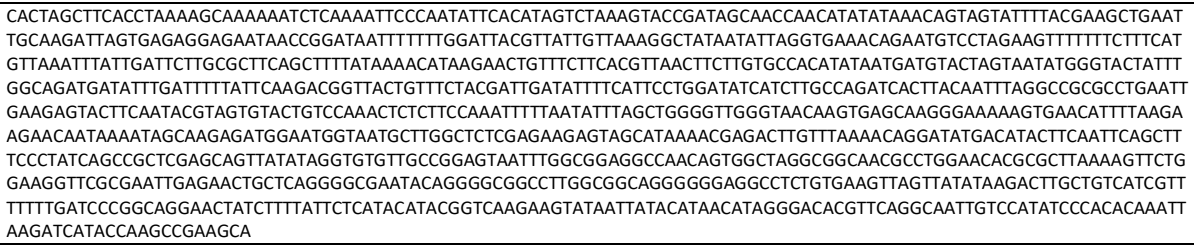 \\
\hline HSP78p-387 & 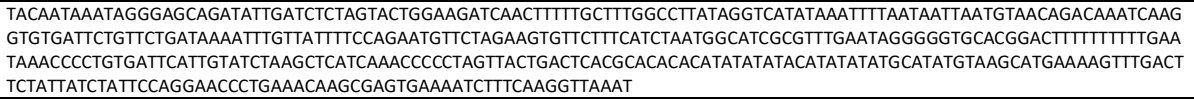 \\
\hline HSP82 $\mathrm{p}_{-858}$ & 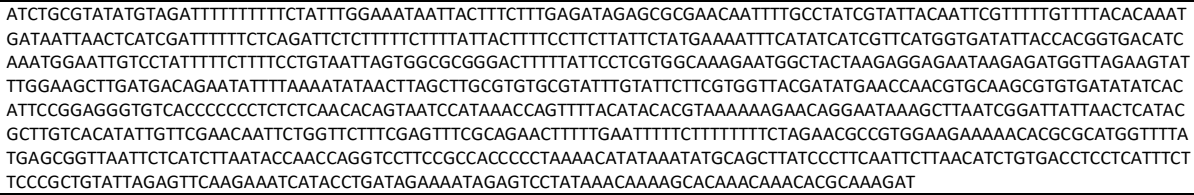 \\
\hline HSP104p-467 & $\begin{array}{l}\text { AAACCTTCTGCACCATTTTAGAAAAAAAGAATCTACCTATTCACTTATTTATTCATTTACTTATTTATTTACATATTTATCATACATATTAACATTGAACCCTCCATCGTG } \\
\text { GTAGTGTTTGCTGTTCCTAACTTTCTTTCGTTGTTCTTGTAGATATATATTTTCCAGAATTTTCTAGAAGGGTATTAATTACAATCTTAAACGTCCATAAGGGGCG } \\
\text { CGATTTTTTGTCAATTTTCAACAGGGGGCCCATCTCAAAGAACTGCAAATTATATCACAGTAAAAGGCAAAGGGGGCAAACTTATGCAACCTGCCAGATTATTATA } \\
\text { TAAGGCATGGTAATCTTGCCTCAATTCCTTCATAATTCGTCCTTTGTCACTTGTTCCTTTTACCCTTGAATCGAATCAGCAATAACAAAGAAAAAAGAAATCAACTACA } \\
\text { CGTACCATAAAATATACAGAATAT }\end{array}$ \\
\hline SIS1p-501 & $\begin{array}{l}\text { CAATCAGAACTCCTTTTTTATATGGATGACAAGACTGCCACGTTGATCGCTGCGCTAATAATATATGGTTGTCGCACTCTTCTATAACATGATCAGTAATAGCTAGTCGT } \\
\text { TACTTTCTGAATCTTAACGTATTGTGTTACCCTCTTGGTIATATGAACGTTCCAGAAACTTCTGGAAAAAGAATGGGATATCCTTTACGGGTTITCAACCACTGTC } \\
\text { AGCACAAACTCCTTGCCAAGGTAGTTCGAGATTTAATATATATTGTATAGATTCTCGTCCCTTTACCGTATGTAATCCCTTTTAAACTTATGCGCTTCTTGTCAAA }\end{array}$ \\
\hline
\end{tabular}




\begin{tabular}{|c|c|}
\hline & $\begin{array}{l}\text { AATTCTAATTAATATTATTTAAGCTCTTACACGAATTCTTTAACGACTTAGAATTGTATAACCATCCAGAAACTCTTCAGAGGATAAAGGATAAGTTGTTTGCATTTIAA } \\
\text { GATTTाTITAATACATTCACATCAATATAATAGAGTATAGTATACAGAACTAATA }\end{array}$ \\
\hline SSA1p -824 & 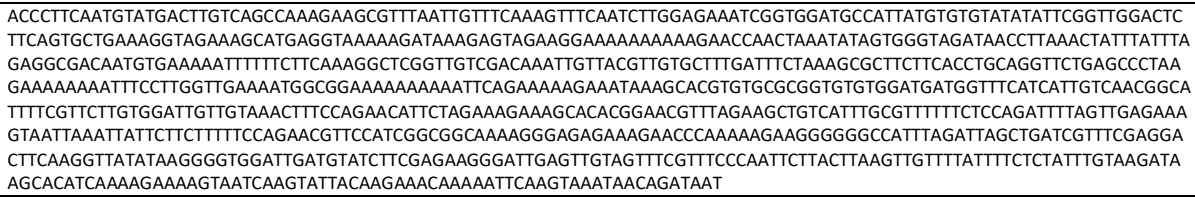 \\
\hline SSA2p-511 & 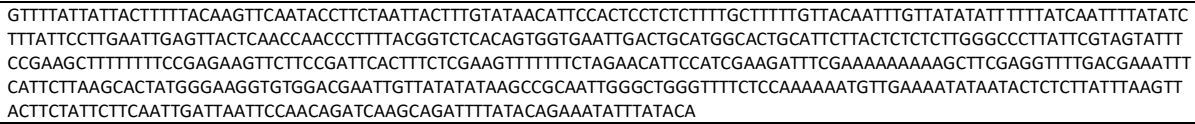 \\
\hline $\begin{array}{l}\text { SSE1 }-587 \\
\end{array}$ & 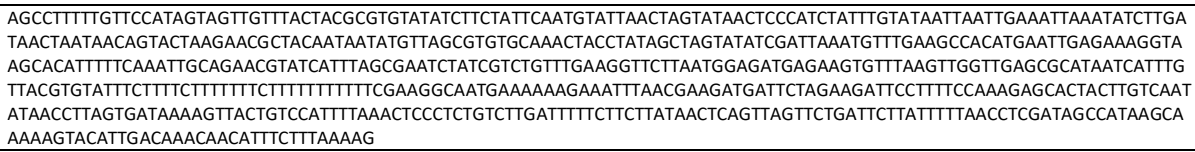 \\
\hline ST/12-246 & $\begin{array}{l}\text { TATCTTCGTGAAGATTCGTGTAGTATGATAGAACATCCAGAAAAAAAATTCAGATTCATCGCTCTCTCTTCGCTTCTCCTCCTTTAAGGAATAAAGAAAAAATCACATA } \\
\text { CATAGATTAAGTAAATAGGATCTGCTAGAAAAATTATATATAGATCAATCATCTATTAAGGTATCTTGTTAAGCCCAAAAGTCTGCTCCCAAATTCCTCACTGTAGCT } \\
\text { ACTAAAACAACCTATACGCAAGAAG }\end{array}$ \\
\hline 1xHSE-GAL1p-231 & $\begin{array}{l}\text { ITTCTAGAACGCCGTGGAAGAACCATAGGATGATAATGCGATTAGTTाTTAGCCTTATTTCTGGGGTAATTAATCAGCGAAGCGATGATTTाTGATCTATTAACAGAT } \\
\text { ATATAAATGGAAAAGCTGCATAACCACTTAACTAATACTTCAACATTTCAGTTGTATACTTCTATTCAAATGTCATAAAAGTATCAACAAAAAATTGTAATATA } \\
\text { CCTCTATACTTAACGTCAAGGAGAAAAAACCCAGTGCAGGT }\end{array}$ \\
\hline 2xHSE-GAL1p-231 & $\begin{array}{l}\text { TाTCTAGAACGTGACGGAAGTTCTAGAACGCCGTGGAAGAACCATAGGATGATAATGCGATTAG TTTTTAGCCTTATTCTGGGGTAATTAATCAGCGAAGCGATG } \\
\text { ATTTTGATCTATAACAGATATATAAATGGAAAGCTGCATAACCACTTAACTAATACTTCAACATTTCAGTTGTATTACTTCTTATTCAAATGTCATAAAAGTAT } \\
\text { CAACAAAAAATTGTAATATACCTCTATACTTAACGTCAAGGAGAAAAACCCAGTGCAGGT }\end{array}$ \\
\hline 4xHSE-GAL1p-231 & 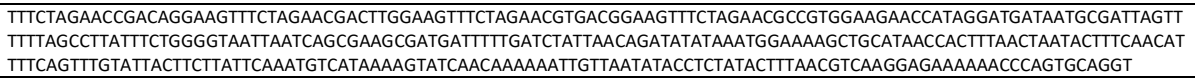 \\
\hline 8xHSE-GALIp-231 & 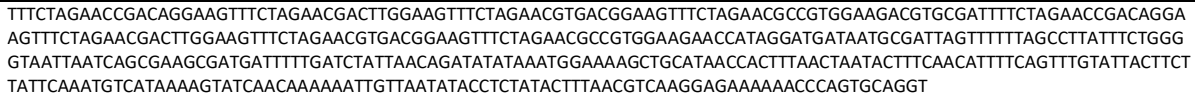 \\
\hline 1xUAS-HSP82p-324:UGFP & 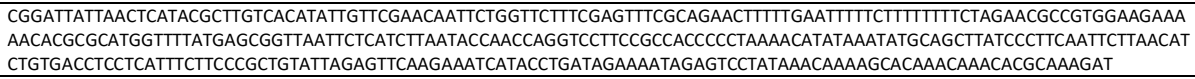 \\
\hline 1XUAS-SIS1p-345:UGFP & $\begin{array}{l}\text { TGAACGTTCCAGAAACTTCTGGAAAAAGAATGGGATATCCTTTTACGGGTTTTTCAACCACTGTCAGCACAAACTCCTTGCCAAGGTAGTTCGAGATTTAATATATATT } \\
\text { GTATAGATTCTCGTCCCTTIACCGTATTGTTAATCCCTTTTAAACTAAGCGCTTCTGTCAAAAATTCTAATTAATATTATTTAAGCTCTACACGAATTCTTTAAACG } \\
\text { ACTTAGAATGTATAACCATCCAGAAACTCTTCAGAGGATAAAGGATAAGTGTTTGCATTTAAGATTTTTTTITAATACATTCACATCAATATAATAGAGTATAGTA } \\
\text { TACAGAACTAATA }\end{array}$ \\
\hline 1xUAS-SSA1p-365:UGFP & 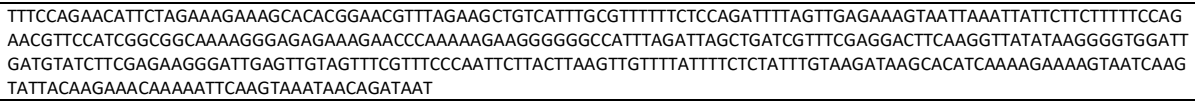 \\
\hline 2xUAS-HSP82p-324:UGFP & 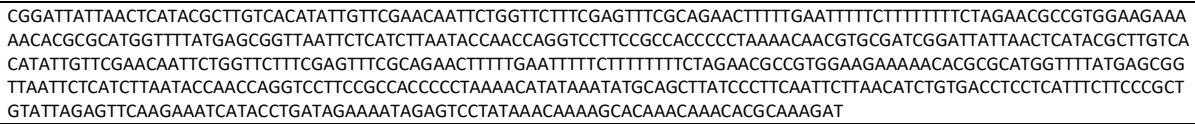 \\
\hline 4xUAS-HSP82p-324:UGFP & 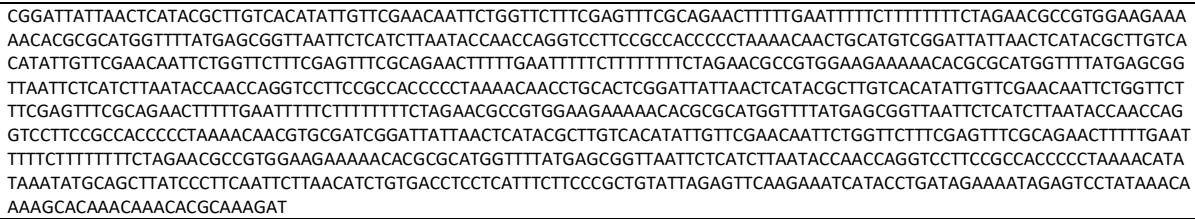 \\
\hline 2xUAS-SIS1p-345:UGFP & 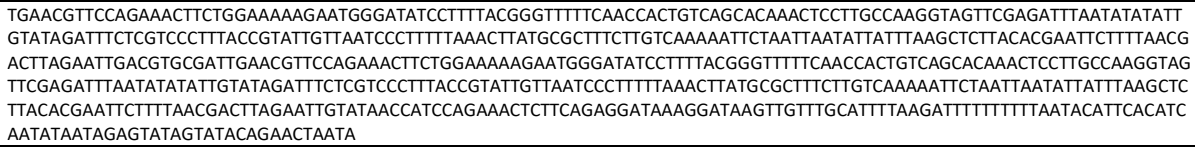 \\
\hline 4xUAS-SIS1P-345:UGFP & 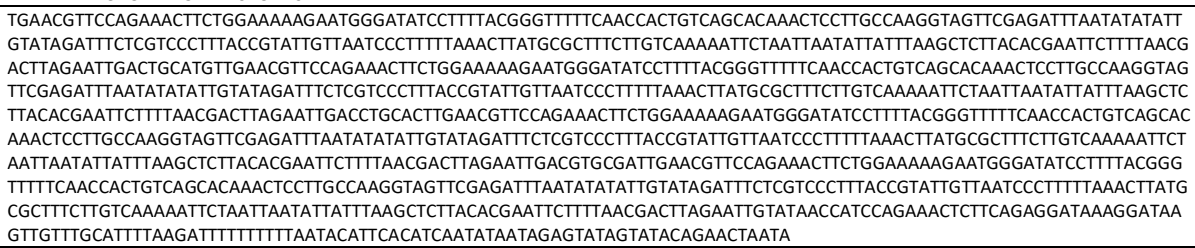 \\
\hline 2xUAS-SSA1p-365:UGFP & 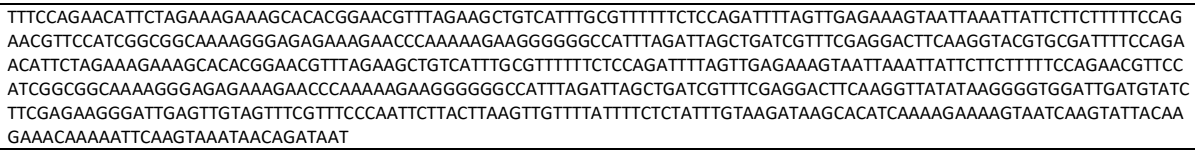 \\
\hline 4xUAS-SSA1p-365:UGFP & 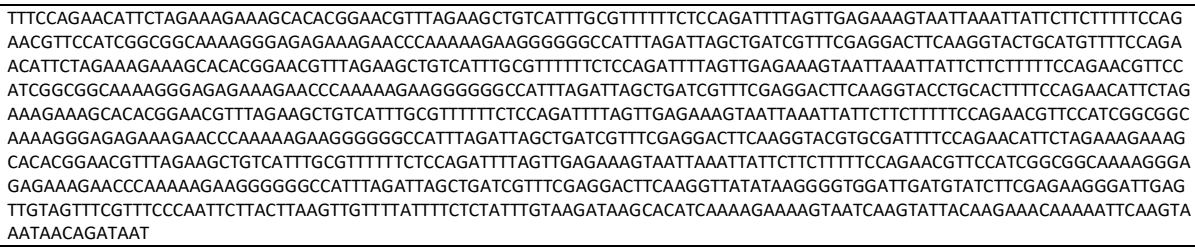 \\
\hline
\end{tabular}


Supplementary Table 6. Sequences of representative plasmids used in this study

\begin{tabular}{|l|l|}
\hline PI & \\
s & Sequence \\
$\mathrm{m}$ & \\
id & \\
\hline
\end{tabular} TAACCCGTACATGCCCAAAATAGGGGGCGGGTTACACAGAATATATAACATCGTAGGTGTCTGGGTGAACAGTTATTCCTGGCATCCACTAAATATAATGGAGCCCGCTITTTAAGCTGGCATCC AGACCCGTACATGCCCAAAATAGGGGGCGGGTTACACAGAATATATAACATCGTAGGTGTCTGGGTGAACAGTITATTCCTGGCATCCACTAAATATAATGGAGCCCCGCTITITAGGCTGGCATCC ATGGAGTGATGCAACCTGCCTGGAGTAAATGATGACACAAGGCAATTGACCCACGCATGTATCTATCTCATTTTCTTACACCTTCTATTACCTTCTGCTCTCTCTGATTTGGAAAAAGCTGAAAAAAA AGGTTGAAACCAGTTCCCTGAAATTATTCCCCTACTTGACTAATAAGTATATAAAGACGGTAGGTATTGATTGTAATTCTGTAAATCTATTTCTTAAACTTCTTAAATTCTACTTTIATAGTTAGTCTTT TITTTAGTTTTAAAACACCAAGAACTTAGTTTCGAATAAACACACATAAACAAACAAAAAAACAATGGGTAGCAGCCACCACCACCACCACCACGGCGGTGGCGGTTCTATGGTTTCTGAACTCATC AAGGAAAACATGCACATGAAACTTTACATGGAAGGTACTGTGAACAATCATCATTTTAAGTGTACATCCGAGGGTGAAGGCAAACCTTACGAAGGAACTCAAACTATGAGAATTAAAGCTGTAGA AGGTGGACCATTACCTTTTGCATTTGATATCTTGGCAACATCATTCATGTATGGGAGCAAGACATTCATAAACCATACTCAAGGTATACCAGACTTTTTCAAACAGAGTTTTCCAGAGGGTTTTACAT GGGAAAGAGTAACAACGTACGAGGATGGAGGTGTATTGACAGCCACTCAAGACACATCACTTCAAGATGGGTGTTTAATCTACAATGTCAAGATTAGAGGCGTCAATTTCCCTTCTAATGGTCCAG TTATGCAGAAAAAGACATTAGGCTGGGAAGCGTCAACCGAAACCCTGTACCCTGCTGATGGTGGCCTAGAAGGCAGAGCTGACATGGCCCTTAAACTGGTTGGTGGAGGGCATCTAATCTGCAAT TTGAAAACCACTTATCGTTCTAAAAAGCCAGCCAAAAACCTAAAGATGCCAGGTGTTTACTACGTCGACCGAAGATTAGAAAGGATTAAAGAGGCTGATAAAGAGACTTATGTTGAACAACACGA AGTGGCAGTGGCTAGATACTGTGATTTGCCATCTAAGTTGGGACACAGAGACGGTGGATCCGGGGGTGGTAGCACTTCTATGAAGATCAAAACGGGCGCAAGAATCTTGGCGCTAAGTGCGTTG ACTACAATGATGTTCTCTGCGTCAGCCCTTGCCAAAATTGAGGAGGGTAAGTTGGTCATCTGGATAAACGGAGACAAGGGCTATAACGGGCTGGCGGAAGTGGGAAAGAAATTCGAGAAGGATA CCGGTATAAAAGTAACAGTTGAACATCCTGATAAATTGGAGGAGAAATTTCCTCAAGTCGCCGCAACGGGGGACGGCCCAGATATCATCTTCTGGGCCCATGATAGATTTGGTGGGTATGCACAA AGCGGCCTACTAGCTGAAATCACACCAGATAAGGCATTCCAAGATAAGTTATATCCATTCACATGGGACGCGGTACGTTATAATGGTAAGCTAATCGCGTACCCTATAGCAGTCGAAGCTCTTTCCC TGATTTACAATAAGGATCTGCTTCCCAACCCACCCAAGACGTGGGAGGAAATACCGGCATTAGATAAGGAGCTAAAAGCGAAGGGAAAATCCGCTTTAATGTTTAACCTGCAAGAACCATATTITA CATGGCCCCTAATCGCCGCCGACGGAGGGTATGCATTTAAGTATGAAAACGGAAAATATGATATAAAAGATGTCGGCGTAGATAACGCGGGAGCCAAGGCGGGGTTAACTTTCCTAGTCGATCTA ATCAAGAACAAGCACATGAACGCCGATACGGATTATTCTATCGCAGAAGCTGCTTTTAATAAAGGAGAAACCGCAATGACTATCAACGGGCCGTGGGCCTGGAGTAACATAGATACGTCTAAGGT GAACTACGGAGTAACCGTTCTGCCGACGTTTAAAGGTCAACCTTCAAAACCCTTCGTCGGCGTGTTAAGCGCAGGGATTAACGCGGCGAGCCCTAATAAGGAACTTGCCAAAGAGTTCCTGGAAA ATTATCTTCTTACCGACGAAGGATTGGAGGCAGTAAATAAAGATAAGCCACTTGGAGCTGTTGCGCTGAAGAGCTATGAGGAGGAGTTAGCGAAGGATCCCCGTATTGCAGCAACAATGGAGAA CGCGCAAAAGGGAGAGATTATGCCGAACATCCCACAGATGTCTGCTTTCTGGTATGCCGTAAGGACGGCCGTCATTAATGCAGCATCCGGCAGGCAGACCGTCGATGAGGCTCTAAAGGACGCAC AGACATGAAGCTTCGAATTTACGTAGCCCAATCTACCACTTTTTTTTTTCATTTTTTAAAGTGTTATACTTAGTTATGCTCTAGGATAATGAACTACTTTTTTTTTTTTTTTTTTACTGTTATCATAAATAT

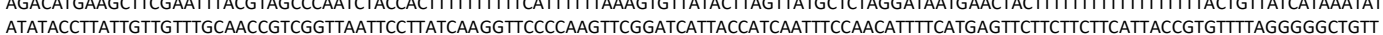
GCAACTCAGAAGTTTGACAGCAAGCAAGTTCATCATTCGAACTAGCCTTATTGTTTTAGTTCAGTGACAGCGAACTGCCGTACTCGATGCTTTATTTCTCACGGTAGAGCGGAAGAACAGATAGGG GCAGCGTGAGAAGAGTTAGAAAGTAAATTTTATCACGTCTGAAGTATTCTTATTCATAGGAAATTTTGCAAGGTTTTTAGCTCAATAACGGGCTAAGTTATATAAGGTGTTCACGCGATTTTCTTG TTATGTATACCTCTCTCTGAGGAATGGTACTACTGTCCTGATGTAGGCTCCTTAAATTGGTGGGCAAGAATAACTTATCGATATTTGTATATTGGTCTTGGAGTTCACCACGTAATGCCTGTTTAA CAATGATTAACTCTATTTAATCCTTTTTGAAAGGCAAAAGAGGTCCCTTGTTCACTTACAACGTTCTTAGCCAAATTCGCTTATCACTTACTACTTCACGATATACAGAAGTAAAAACATATAAAAAG ATGTCTGTTTGTTTAGCCATCACAAAAGGTATCGCAGATTAAGACCTCAGCGCGGCCGCAAATTTAAATAAAATGAAGTGAAGTTCCTATACTTTCTAGAGAATAGGAACTTCTATAGTGAGTCGAA CGAGATTITTTTCTAATTCTCTTTACAAACTAGAAATATGTATATACAAAAAATCATAAATAATAGATGAATAGTTTAATATAGGTGTCATCAATCGAAAAAGCAACGTATCTTATTAAAGT GCGTTGCTTTTTCTCATTTATAAGGTTAAATAATTCTCATATATCAAGCAAAGTGACAGGCGCCCTTAAATATTCTGACAAATGCTCTTTCCCTAAACTCCCCCCATAAAAAAACCCGCCGAAGCGG GTTITACGTTATTTGCGGATTAACGATTACTCGTTATCAGAACCGCCCAGGGGGCCCGAGCTTAAGACTGGCCGTCGTITTACAACACAGAAAGAGTTTGTAGAAACGCAAAAAGGCCATCCGTC AGGGGCCTTCTGCTTAGTTGATGCCTGGCAGTCCCTACTCTCGCCTCCGCTTCCTCGCTCACTGACTCGCTGCGCTCGGTCGTCGGCTGCGGCGAGCGGTATCAGCTCACTCAAAGGCGGTAA AGGGGCCTTCTGCTTAGTTTGATGCCTGGCAGTTCCCTACTCTCGCCTTCCGCTTCCTCGCTCACTGACTCGCTGCGCTCGGTCGITCGGCTGCGGCGAGCGGTATCAGCTCACTCAAAGGCGGTAA

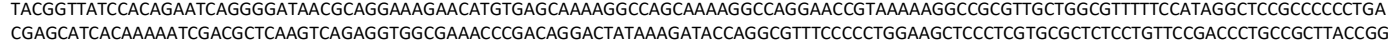
ATACCTGTCCGCCTTTCTCCCTTCGGGAAGCGTGGCGCTTTCTCATAGCTCACGCTGTAGGTATCTCAGTTCGGTGTAGGTCGTTCGCTCCAAGCTGGGCTGTGTGCACGAACCCCCCGTTCAGCCC GACCGCTGCGCCTTATCCGGTAACTATCGTCTTGAGTCCAACCCGGTAAGACACGACTTATCGCCACTGGCAGCAGCCACTGGTAACAGGATTAGCAGAGCGAGGTATGTAGGCGGTGCTACAGA GTTCTTGAAGTGGTGGGCTAACTACGGCTACACTAGAAGAACAGTATTTGGTATCTGCGCTCTGCTGAAGCCAGTTACCTTCGGAAAAAGAGTTGGTAGCTCTTGATCCGGCAAACAAACCACCGC TGGTAGCGGTGGTTTITTGTTTGCAAGCAGCAGATTACGCGCAGAAAAAAAGGATCTCAAGAAGATCCTTTGATCTTTTCTACGGGGTCTGACGCTCAGTGGAACGACGCGCGCGTAACTCACG TAAGGGATTTGGGTCATGAGCTTGCGCCGTCCCGTCAAGTCAGCGTAATGCTCTGCTTTTACCAATGCTTAATCAGTGAGGCACCTATCTCAGCGATCTGTCTATTTCGTTCATCCATAGTTGCCTGA CTCCCCGTCGTGTAGATAACTACGATACGGGAGGGCTTACCATCTGGCCCCAGCGCTGCGATGATACCGCGAGAACCACGCTCACCGGCTCCGGATTTATCAGCAATAAACCAGCCAGCCGGAAG GGCCGAGCGCAGAAGTGGTCCTGCAACTTTATCCGCCTCCATCCAGTCTATTAATTGTTGCCGGGAAGCTAGAGTAAGTAGTTCGCCAGTTAATAGTTTGCGCAACGTTGTTGCCATCGCTACAGG CATCGTGGTGTCACGCTCGTCGTTTGGTATGGCTTCATTCAGCTCCGGTTCCCAACGATCAAGGCGAGTIACATGATCCCCCATGTTGTGCAAAAAAGCGGTIAGCTCCTTCGGTCCTCCGATCGTT GTCAGAAGTAAGTTGGCCGCAGTGTTATCACTCATGGTTATGGCAGCACTGCATAATTCTCTTACTGTCATGCCATCCGTAAGATGCTTTTCTGTGACTGGTGAGTACTCAACCAAGTCATTCTGAG AATAGTGTATGCGGCGACCGAGTTGCTCTTGCCCGGCGTCAATACGGGATAATACCGCGCCACATAGCAGAACTTTAAAAGTGCTCATCATTGGAAAACGTTCTTCGGGGCGAAAACTCTCAAGG ATCTTACCGCTGTTGAGATCCAGTTCGATGTAACCCACTCGTGCACCCAACTGATCTTCAGCATCTTTTACTTTCACCAGCGTTTCTGGGTGAGCAAAAACAGGAAGGCAAAATGCCGCAAAAAAGG GAATAAGGGCGACACGGAAATGTTGAATACTCATATTCTTCCTTTTTCAATATTATTGAAGCATTTATCAGGGTTATTGTCTCATGAGCGGATACATATTTGAATGTATTTAGAAAAATAAACAAATA GGGGTCAGTGTTACAACCAATTAACCAATTCTGAACATTATCGCGAGCCCATTTATACCTGAATATGGCTCATAACACCCCTTGTTTGCCTGGCGGCAGTAGCGCGGTGGTCCCACCTGACCCCATG CCGAACTCAGAAGTGAAACGCCGTAGCGCCGATGGTAGTGTGGGGACTCCCCATGCGAGAGTAGGGAACTGCCAGGCATCAAATAAAACGAAAGGCTCAGTCGAAAGACTGGGCCTTTCGCCCG GGCTAATTATGGGGTGTCGCCCTTATTCGACTCTATAGTGAAGTTCCTATTCTCTAGAAAGTATAGGAACTTCTGAAGTGGGGATTTAAATGCGGCCGCGCTGAGGGTTTAATCAATCTGGCGGCTT GAGTTCTCAACATGTTTTATTTTTTACTTATATTGCTGGTAGGGTAAAAAAATATAACTCCTAGGAATAGGTTGTCTATATGTTTTTGTCTTGCTTCTATAATTGTAACAAACAAGGAAAGGGAAAAT ACTGGGTGTAAAAGCCATTGAGTCAAGTTAGGTCATCCCTTTTATACAAAATTTTTCAATTTTTTTTCCAAGATTCTTGTACGATTAATTATTTTTTTTTTGCGTCCTACAGCGTGATGAAAATTTCGCC TGCTGCAAGATGAGCGGGAACGGGCGAAATGTGCACGCGCACAACTTACGAAACGCGGATGAGTCACTGACAGCCACCGCAGAGGTTCTGACTCCTACTGAGCTCTATTGGAGGTGGCAGAACC GGTACCGGAGGAGACCGCTATAACCGGTTTGAATTTATTGTCACAGTGTCACATCAGC

TTTCCAGAACATTCTAGAAAGAAAGCACACGGAACGTTTAGAAGCTGTCATTTGCGTTTTTTCTCCAGATTTTAGTTGAGAAAGTAATTAAATTATTCTTCTTTTTCCAGAACGTTCCATCGGCGGCA AAAGGGAGAGAAAGAACCCAAAAAGAAGGGGGGCCATTTAGATTAGCTGATCGTTTCGAGGACTTCAAGGTACTGCATGTTTTCCAGAACATTCTAGAAAGAAAGCACACGGAACGTTTAGAAG CTGTCATTTGCGTTTTTCTCCAGATTTTAGTTGAGAAAGTAATTAAATTATTCTTCTTTTTCCAGAACGTTCCATCGGCGGCAAAAGGGAGAGAAAGAACCCAAAAAGAAGGGGGGCCATTTAGAT TAGCTGATCGTTTCGAGGACTTCAAGGTACCTGCACTTTTCCAGAACATTCTAGAAAGAAAGCACACGGAACGTTTAGAAGCTGTCATTTGCGTTTTTTCTCCAGATTTAGTTGAGAAAGTAATTAA ATTATTCTTCTTTTTCCAGAACGTTCCATCGGCGGCAAAAGGGAGAGAAAGAACCCAAAAAAGAAGGGGGGCCATTTAGATTAGCTGATCGTTTCGAGGACTTCAAGGTACGTGCGATTTTCCAGAA CATTCTAGAAAGAAAGCACACGGAACGTTTAGAAGCTGTCATTTGCGTTTTTCTCCAGATTTIAGTTGAGAAAGTAATTAAATTATTCTTCTTTTTCCAGAACGTICCATCGGCGGCAAAAGGGAG AGAAAGAACCCAAAAAGAAGGGGGGCCATTTAGATTAGCTGATCGTTTCGAGGACTTCAAGGTTATATAAGGGGTGGATTGATGTATCTTCGAGAAGGGATTGAGTTGTAGTTTCGTTTCCCAAT TCTTACTTAAG TTGTTTTATTTTCTCTATTTGTAAGATAAGCACATCAAAAGAAAAGTAATCAAGTATTACAAGAAACAAAAATTCAAGTAAATAACAGATAATACTTGTCATAAAACAATGTCTAAA GGTGAAGAATTATTCACTGGTGTTGTCCCAATTTTGGTTGAATTAGATGGTGATGTTAATGGTCACAAATTTTCTGTCTCCGGTGAAGGTGAAGGTGATGCTACTTACGGTAAATTGACCTTAAAAT TTATTTGTACTACTGGTAAATTGCCAGTTCCATGGCCAACCTTAGTCACTACTTTCGGTTATGGTGTTCAATGTTTTGCGAGATACCCAGATCATATGAAACAACATGACTTTTTCAAGTCTGCCATGC CAGAAGGTTATGTTCAAGAAAGAACTATTTITTTCAAAGATGACGGTAACTACAAGACCAGAGCTGAAGTCAAGTTTGAAGGTGATACCTTAGTTAATAGAATCGAATTAAAAGGTATTGATTTTA AAGAAGATGGTAACATTTTAGGTCACAAATTGGAATACAACTATAACTCTCACAATGTTTACATCATGGCTGACAAACAAAAGAATGGTATCAAAGTTAACTTCAAAATTAGACACAACATTGAAGA TGGTTCTGTTCAATTAGCTGACCATTATCAACAAAATACTCCAATTGGTGATGGTCCAGTCTTGTTACCAGACAACCATTACTTATCCACTCAATCTGCCTTATCCAAAGATCCAAACGAAAAGAGAG ACCACATGGTCTTGTTAGAATTTGTTACTGCTGCTGGTATTACCCATGGTATGGATGAATTGTACAAATGAACACGCGATATCCGCTCTAACCGAAAAGGAAGGAGTTAGACAACCTGAAGTCTAG GTCCCTATTTATTTTTTTATAGTTATGTTAGTATTAAGAACGTTATTTATATTTCAAATTTTTCTTTTTTTTCTGTACAGACGCGTGTACGCATGTAACATTATACTGAAAACCTTGCTTGAGAAGGTTT GGGACGCTCGAAGATCGCGTACCCAATTCGCCCTATAGTGAGTCGTATTACGCGCGCTCACTGGCCGTCGTTTTACAACGTCGTGACTGGGAAAACCCTGGCGTTACCCCTGCAGGACTAGTGCTG AGGCATTAATACATGGGAAGATTCGCTTTTTTTTTTGAATTACAATAGTATGTCTGATGTCTGCAAGAAGTAACAGGCGTGTGCACAAGAATACGTGTGTGTGCGTAAGCGTATGCACTGGTGGC ATAACTTATCTAAGAAGTATATATCACTGACATAGAAATGTAGATATACAGGTATTTTTCTCGATAATCGATAAAAATCTCGTCGCGCTGAACCAAACTTGGTGGTTACGGAGAGTTTTCTCTCATC ATTACTGTCTTTCGCATTGATTTCCCCTTTGACCGATAAAATCCCTTGGATTCATAAGATTAAACAAAGAGGTGATCAAAGAGAACCCTGTGAAAGTTTATGTTTATAACCGGGCATAAAGTGAACT AGACACTTTCAAGAAGCCAACCAAAGCATGAGTAACGAAGCTTACCAGCATGATCATACCGTAAATCCTCACCAGATTAAGACCTCAGCGCGGCCGCAAATTTAAATAAAATGAAGTGAAGTTCCT ATACTTTCTAGAGAATAGGAACTTCTATAGTGAGTCGAATAAGGGCGACACAAAATTTATTCTAAATGCATAATAAATACTGATAACATCTTATAGTTTGTATTATATTTTGTATTATCGTTGACATGT ATAATTTTGATATCAAAAACTGATTTTCCCTTTATTATTTTCGAGATTTATTTTCTTAATTCTCTTTAACAAACTAGAAATATTGTATATACAAAAAATCATAAATAATAGATGAATAGTTTAATTATAG GTGTTCATCAATCGAAAAAGCAACGTATCTTATTTAAAGTGCGTTGCTTTTTTCTCATTTATAAGGTTAAATAATTCTCATATATCAAGCAAAGTGACAGGCGCCCTTAAATATTCTGACAAATGCTCT TTCCCTAAACTCCCCCCATAAAAAAACCCGCCGAAGCGGGTTTTIACGTTATTTGCGGATTAACGATTACTCGTTATCAGAACCGCCCAGGGGGCCCGAGCTTAAGACTGGCCGTCGTTTTACAACA GCTGCGGCGAGCGGTATCAGCTCACTCAAAGGCGGTAATACGGTTATCCACAGAATCAGGGGATAACGCAGGAAAGAACATGTGAGCAAAAGGCCAGCAAAAGGCCAGGAACCGTAAAAAGGC CGCGTGCTGGCGTITTCCATAGGCTCCGCCCCCCTGACGAGCATCACAAAAATCGACGCTCAAGTCAGAGGTGGCGAAACCCGACAGGACTATAAAGATACCAGGCGTTTCCCCCTGGAAGCTC CGCGTTGCTGGCGTTTTCCATAGGCTCCGCCCCCCTGACGAGCATCACAAAAATCGACGCTCAAGTCAGAGGTGGCGAAACCCGACAGGACTATAAAGATACCAGGCGTTTCCCCCTGGAAGCTC
CCTCGTGCGCTCTCCTGTTCCGACCCTGCCGCTTACCGGATACCTGTCGCCTTTCTCCCTTCGGGAAGCGTGGCGCTTTCTCATAGCTCACGCTGTAGGTATCTCAGTTCGGTGTAGTCGTTCGCT CCTCGTGCGCTCTCCTGTTCCGACCCTGCCGCTTACCGGATACCTGTCCGCCTTTCTCCCTTCGGGAAGCGTGGCGCTTTCTCATAGCTCACGCTGTAGGTATCTCAGTTCGGTGTAGGTCGTTCGCT
CCAAGCTGGGCTGTGTGCACGAACCCCCCGTTCAGCCCGACCGCTGCGCCTTATCCGGTAACTATCGTCTTGAGTCCAACCCGGTAAGACACGACTTATCGCCACTGGCAGCAGCCACTGGTAACA GGATTAGCAGAGCGAGGTATGTAGGCGGTGCTACAGAGTTCTTGAAGTGGTGGGCTAACTACGGCTACACTAGAAGAACAGTATTTGGTATCTGCGCTCTGCTGAAGCCAGTTACCTTCGGAAAA AGAGTGGTAGCTCTTGATCCGGCAAACAAACCACCGCTGGTAGCGGTGGTTTTTTTGTTTGCAAGCAGCAGATTACGCGCAGAAAAAAAGGATCTCAAGAAGATCCTTTGATCTTTTCTACGGGG 


\begin{abstract}
TCTGACGCTCAGTGGAACGACGCGCGCGTAACTCACGTTAAGGGATTTGGTCATGAGCTTGCGCCGTCCCGTCAAGTCAGCGTAATGCTCTGCTTTTACCAATGCTTAATCAGTGAGGCACCTATC TCAGCGATCTGTCTATTTCGTTCATCCATAGTGGCTGACTCCCCGTCGTGTAGATAACTACGATACGGGAGGGCTACCATCTGGCCCCAGCGCTGCGATGATACCGCGAGAACCACGCTCACCGG CTCCGGATTATCAGCAATAAACCAGCCAGCCGGAAGGGCGAGCGCAGAAGTGGTCCTGCAACTTTATCCGCCTCCATCCAGTCTATTAATTGTTGCCGGGACCTAGAGTAAGTAGTTCGCCAG TTAATAGTTGCOACGTTGTTGCCATCGCTACAGGCATCGTGGTGTCACGCTCGTCGTTTGGTATGGCTCATTCAGCTTCGGTTCCCAACGATCAAGGCGAGTTACATGATCCCCCATGTTGTGC AAAAAGT TGTGACTGGTGAGTACTCAACCAAGTCATTCTGAGATAGTGTATA T. TGGAAAACGTCTICGGGCGAAAACTCTCAAGGATCTACCGCTGTTCAGTCCAGTICGATGTAACCCACTCGTGCACCCAACTGATCTTCAGCATCTITACTITCACCAGCGTICTGGGTGAG CAAAAACAGGAAGGCAAAATGCCGCAAAAAAGGGAATAAGGGCGACACGGAAATGTTGAATACTCATATTCTTCCTTTTTCAATATTATTGAAGCATTTATCAGGGTTATTGTCTCATGAGCGGAT ACATATTTGAATGTATTTAGAAAAATAAACAAATAGGGGTCAGTGTTACAACCAATTAACCAATTCTGAACATTATCGCGAGCCCATTTATACCTGAATATGGCTCATAACACCCCTTGTTTGCCTGG CGGCAGTAGCGCGGTGGTCCCACCTGACCCCATGCCGAACTCAGAAGTGAAACGCCGTAGCGCCGATGGTAGTGTGGGGACTCCCCATGCGAGAGTAGGGAACTGCCAGGCATCAAATAAAACG AAAGGCTCAGTCGAAAGACTGGGCCTTTCGCCCGGGCTAATTATGGGGTGTCGCCCTTATTCGACTCTATAGTGAAGTTCCTATTCTCTAGAAAGTATAGGAACTTCTGAAGTGGGGATTTAAATG CGGCCGCGCTGAGGGTTTAATCCCAAAGCTAAGAGTCCCATTTTATTCTTCTATATGTATATTTCGATACTCTAAACCACCCTACAATGTAGCCCTATACTAAATCTGCTCAATTTCAGCTTCTACAA GTGACTGAGCCACGTGAAGATCCAACTACTCCAGCACAACGATTCAATATAATCGATTGCTCCACTCATAAGAGGCAAGACAAGCTTCAACTTTGGTAAGCGCCGTTATAAACAGGGA AGATCTCGT AGATGTCCTIGTCAAGGGAGGCACAGAGCATGGCCAATIGGCAAATTGCATAATCGATTGCTGAGTGAAAATGAAAAAGCATIGTAGTAGAGTCGGCTCACTGAAAAACCGGGGAGGACGAAAA GGTTTCCAGCCACAGTTGTAGTCACGTGCGCGCCATGCTGACTAATGGCAGCCGTCGTTGGGCAGAAGAGAATTAGTATGGTACAGGATACGCTAATTGCGCTCCAACTACCAAGGTTGTTGAGG GAACACTGGGGCAATAGGCTGTCGCCATTCAAGAGCAGAGACACTTT
\end{abstract}

Supplementary Table 7. TFs that regulate genes upregulated upon mKate2-LipPks1 expression

\begin{tabular}{|c|c|c|c|}
\hline Systematic Name & Gene & Regulators & Evidence \\
\hline YAL005C & SSA1 & Hsf1p & Doc \\
\hline YAL005C & & Rap1p & Doc \\
\hline YAL005C & & Msn2p & Doc \\
\hline YAL005C & & Msn4p & Doc \\
\hline YALO05C & & Skn7p & Doc \\
\hline YAL005C & & Yrr1p & Doc \\
\hline YAL005C & & Fkh1p & Doc \\
\hline YPL240C & HSP82 & Hsf1p & Doc \\
\hline YPL240C & & Msn2p & Doc \\
\hline YPL240C & & $M s n 4 p$ & Doc \\
\hline YPL240C & & Fkh1p & Doc \\
\hline YPL240C & & Yap1p & Doc \\
\hline YPL240C & & Pho2p & Doc \\
\hline YPL240C & & Sum1p & Doc \\
\hline YDR258C & HSP78 & Hsf1p & Doc \\
\hline YDR258C & & Msn2p & Doc \\
\hline YDR258C & & Msn4p & Doc \\
\hline YDR258C & & Gcn $4 p$ & Doc \\
\hline YDR258C & & Fkh1p & Doc \\
\hline YDR258C & & Yap1p & Doc \\
\hline YDR214W & AHA1 & Hsf1p & Doc \\
\hline YDR214W & & Spt23p & Doc \\
\hline YDR214W & & Msn2p & Doc \\
\hline YDR214W & & Msn4p & Doc \\
\hline YDR214W & & Gcn $4 p$ & Doc \\
\hline YDR214W & & Yrr1p & Doc \\
\hline YLL026W & HSP104 & Hsf1p & Doc \\
\hline YLL026W & & Msn2p & Doc \\
\hline YLL026W & & Msn4p & Doc \\
\hline YLL026W & & Fkh1p & Doc \\
\hline YLL026W & & Yap1p & Doc \\
\hline YLL026W & & Pdr1p & Doc \\
\hline YLL026W & & Sko1p & Doc \\
\hline YLL024C & $S S A 2$ & Hsf1p & Doc \\
\hline
\end{tabular}




\begin{tabular}{|c|c|c|c|}
\hline YOR027W & STI1 & Hsf1p & Doc \\
\hline YOR027W & & Spt23p & Doc \\
\hline YOR027W & & Msn2p & Doc \\
\hline YOR027W & & Msn4p & Doc \\
\hline YOR027W & & Abf1p & Doc \\
\hline YNL007C & SIS1 & Hsf1p & Doc \\
\hline YNL007C & & Spt23p & Doc \\
\hline YNL007C & & Msn2p & Doc \\
\hline YNL007C & & Msn4p & Doc \\
\hline YNL007C & & Skn7p & Doc \\
\hline YDR171W & HSP42 & Hsf1p & Doc \\
\hline YDR171W & & Msn2p & Doc \\
\hline YDR171W & & Msn4p & Doc \\
\hline YDR171W & & Rtg3p & Doc \\
\hline YDR171W & & Skn7p & Doc \\
\hline YDR171W & & Ste12p & Doc \\
\hline YDR171W & & Tec1p & Doc \\
\hline YMR186W & HSC82 & Hsf1p & Doc \\
\hline YMR186W & & Rpn4p & Doc \\
\hline YMR186W & & Sfp1p & Doc \\
\hline YMR186W & & Msn4p & Doc \\
\hline YMR186W & & Ino2p & Doc \\
\hline YMR186W & & Ino4p & Doc \\
\hline YMR186W & & Bas1p & Doc \\
\hline YMR186W & & Reb1p & Doc \\
\hline YMR186W & & Yap6p & Doc \\
\hline YLR216C & CPR6 & Hsf1p & Doc \\
\hline YPL106C & SSE1 & Hsf1p & Doc \\
\hline YBR101C & FES1 & Hsf1p & Doc \\
\hline YBR101C & & Rpn4p & Doc \\
\hline YBR101C & & Spt23p & Doc \\
\hline YNL281W & $\mathrm{HCH} 1$ & Hsf1p & Doc \\
\hline YNL281W & & Msn2p & Doc \\
\hline YOR298C-A & MBF1 & Hsf1p & Doc \\
\hline YOR298C-A & & Rap1p & Doc \\
\hline YOR298C-A & & Msn2p & Doc \\
\hline YOR298C-A & & Cbf1p & Doc \\
\hline YFL016C & MDJ1 & Hsf1p & Doc \\
\hline YFL016C & & Spt23p & Doc \\
\hline YFL016C & & Msn2p & Doc \\
\hline YFL016C & & Msn4p & Doc \\
\hline YFL016C & & Yrr1p & Doc \\
\hline YFL016C & & Adr1p & Doc \\
\hline YFL016C & & Ino2p & Doc \\
\hline YFL016C & & Ino4p & Doc \\
\hline YNL064C & YDJ1 & Hsf1p & Doc \\
\hline
\end{tabular}




\begin{tabular}{|c|c|c|c|}
\hline YNL064C & & Msn4p & Doc \\
\hline YNL064C & & Cbf1p & Doc \\
\hline YJL034W & KAR2 & Hsf1p & Doc \\
\hline YJL034W & & Sfp1p & Doc \\
\hline YJL034W & & Hac1p & Doc \\
\hline YER103W & SSA4 & Hsf1p & Doc \\
\hline YER103W & & Msn2p & Doc \\
\hline YER103W & & Msn4p & Doc \\
\hline YER103W & & Ste12p & Doc \\
\hline YER103W & & Yrr1p & Doc \\
\hline YER103W & & $\operatorname{Cin} 5 p$ & Doc \\
\hline YER103W & & Ecm22p & Doc \\
\hline YLR259C & HSP60 & Hsf1p & Doc \\
\hline YHR216W & IMD2 & Rap1p & Doc \\
\hline YHR216W & & Ino4p & Doc \\
\hline YGR211W & ZPR1 & Hsf1p & Doc \\
\hline YBR025C & OLA1 & Hsf1p & Doc \\
\hline YBR025C & & Msn2p & Doc \\
\hline YOR020C & HSP10 & Hsf1p & Doc \\
\hline YJR045C & SSC1 & Hsf1p & Doc \\
\hline YJR045C & & Msn2p & Doc \\
\hline YJR045C & & Skn7p & Doc \\
\hline YOR007C & SGT2 & Hsf1p & Doc \\
\hline YOR007C & & Rpn4p & Doc \\
\hline YOR007C & & Msn2p & Doc \\
\hline YDL082W & RPL13A & Fkh2p & Doc \\
\hline YDL082W & & Ifh1p & Doc \\
\hline YDL082W & & Rap1p & Doc \\
\hline YDL082W & & Sfp1p & Doc \\
\hline YBR082C & UBC4 & Hsf1p & Doc \\
\hline YBR082C & & Ste12p & Doc \\
\hline YBR082C & & Cbf1p & Doc \\
\hline YBR082C & & Crz1p & Doc \\
\hline YDL064W & UBC9 & NA & - \\
\hline YLR421C & RPN13 & NA & - \\
\hline YGR169C-A & $\mathrm{LSO}_{2}$ & NA & - \\
\hline
\end{tabular}

Supplementary Table 8. Over-represented TFBS in genes upregulated upon mKate2LipPks1 expression

\begin{tabular}{|l|l|r|r|}
\hline YeTFaSCo Motif ID & Gene Name & \multicolumn{2}{|l|}{$\begin{array}{l}\text { p-value } \\
\text { (uncorrected) }\end{array}$} \\
\hline YGL073W_411 & HSF1 & $1.56 \mathrm{E}-11$ & 0.862602 \\
\hline YGL073W_1461 & HSF1 & $5.28 \mathrm{E}-11$ & 0.852927 \\
\hline YGL073W_615 & HSF1 & $7.86 \mathrm{E}-10$ & 0.830609 \\
\hline YGL073W_476 & HSF1 & 0.000129 & 0.705831 \\
\hline YPR196W_861 & & 0.004195 & 0.653971 \\
\hline
\end{tabular}




\begin{tabular}{|l|l|l|l|} 
& & & \\
YOR140W_605 & SFL1 & 0.004523 & 0.652686 \\
\hline YMR072W_541 & ABF2 & 0.006137 & 0.647371 \\
\hline YBR033W_517 & EDS1 & 0.006263 & 0.647011 \\
\hline YCR065W_570 & HCM1 & 0.00688 & 0.35466 \\
\hline YMR042W_1483 & ARG80 & 0.008542 & 0.641428 \\
\hline
\end{tabular}

\section{Supplementary References}

1. DiCarlo, J. E., Norville, J. E., Mali, P., Rios, X., Aach, J., \& Church, G. M. (2013). Genome engineering in Saccharomyces cerevisiae using CRISPR-Cas systems. Nucleic Acids Res. 41(7), 4336-4343.

2. Zhang, J., Petersen, S. D., Radivojevic, T., Ramirez, A., Perez, A., Abeliuk, E., Sanchez, B.J., Costello, Z., Chen, Y., Fero, M., Garcia-Martin, H, Nielsen, J., Keasling, J.D., Jensen, M.K. (2020). Predictive engineering and optimization of tryptophan metabolism in yeast through a combination of mechanistic and machine learning models. Nat. Commun. 11 (1), 4880. 